\title{
Numerical approximations of the Mumford-Shah functional for unit vector fields
}

\author{
JONAS HAEHNLE \\ Mathematisches Institut, Universität Tübingen, \\ Auf der Morgenstelle 10, D-72076 Tübingen, Germany \\ E-mail: haehnle@na.uni-tuebingen.de
}

[Received 7 May 2009 and in revised form 10 June 2011]

Two numerical approximation schemes for minimising the Mumford-Shah functional for unit vector fields are proposed, analysed, and compared. The first uses a projection strategy, the second a penalisation strategy to enforce the sphere constraint. Both schemes are then applied to the segmentation of colour images using the Chromaticity and Brightness colour model.

\section{Introduction}

For $\Omega \subset \mathbb{R}^{d}$, and $\gamma, \alpha, \lambda$ positive constants, we are interested in numerically minimising the following weak version of the Mumford-Shah energy functional:

$$
G(\mathbf{u}):=\frac{\gamma}{2} \int_{\Omega}|\nabla \mathbf{u}|^{2} \mathrm{~d} \mathbf{x}+\alpha \mathcal{H}^{d-1}\left(S_{\mathbf{u}}\right)+\frac{\lambda}{2} \int_{\Omega}|\mathbf{u}-\mathbf{g}|^{2} \mathrm{~d} \mathbf{x}
$$

with $\mathbf{u}, \mathbf{g} \in \operatorname{GSBV}\left(\Omega, \mathbb{R}^{m}\right)$, and $|\mathbf{u}|^{2}=1$ a.e. (see Section 2 for definitions). This is a prototype problem for studying interesting effects with applications in image processing (see e.g. [43, 44, 8, $10,19,50,7]$ ), and liquid crystal theory (see e.g. [39, 42, 21, 51, 1, 6, 16]).

We are sometimes going to refer to functional (1.1) as the "Mumford-Shah" functional. It is, in fact, a version (for sphere-valued functions) of a functional proposed by De Giorgi, Carriero, and Leaci in [27] (for scalar functions) as a weak formulation of the original functional proposed by Mumford and Shah in [43] for greyscale image segmentation,

$$
E(u, K):=\frac{\gamma}{2} \int_{\Omega \backslash K}|\nabla u|^{2} \mathrm{~d} \mathbf{x}+\alpha \mathcal{H}^{d-1}(K)+\frac{\lambda}{2} \int_{\Omega}(u-g)^{2} \mathrm{~d} \mathbf{x},
$$

with $g \in L^{2}(\Omega)$, which is to be minimised for all closed sets $K \subset \Omega$ and functions $u \in H^{1}(\Omega \backslash K)$. It is shown in [27] that the two problems are essentially equivalent.

The goal of image segmentation is to partition images into meaningful regions, which is often done by finding the edges which bound these regions, and which are in our case identified with the set $K$. The first term in (1.2) ensures smoothness of $u$ outside of $K$, the second one ensures that there are not too many edges, and the last term ensures that the segmented image $u$ does not deviate too much from the original one $g$.

A more concrete motivation for studying functional (1.1), therefore is colour image segmentation in the Chromaticity and Brightness $(C B)$ colour model, where the chromaticity (colour information) is represented by an $\mathbb{S}^{m-1}$-valued function (usually $m=3$ ) on the image domain $\Omega$. The brightness, represented by a function $b: \Omega \rightarrow[0,1]$, can be separately treated just like a 
greyscale image. It has been suggested that this model is well-suited for colour image processing. Osher and Vese [44] studied $p$-harmonic flows to the sphere ( $p \geqslant 1$, in particular $p \in\{1,2\})$, and applied them to image chromaticity, for example; other sources include $[19,50,7]$ and references therein.

The name free discontinuity problems was introduced by De Giorgi in [24] for variational problems like (1.2), which consist in minimising a functional with volume and surface terms, depending on a closed set $K$ and a function $u$ (usually smooth outside $K$ ). Other early sources include [26, 25]. Weak formulations like (1.1) allow one to prove existence of solutions (see [27] for the scalar and [17] for the sphere-valued case), but still require the computation of geometric properties of the unknown set of discontinuity boundaries.

Therefore, Ambrosio and Tortorelli introduced an elliptic approximation in [3, 4], whose vectorial version, if defined for sphere-valued functions, is to minimise

$$
\begin{aligned}
A T_{\varepsilon}(\mathbf{u}, s):= & \frac{\gamma}{2} \int_{\Omega}\left(s^{2}+k_{\varepsilon}\right)|\nabla \mathbf{u}|^{2} \mathrm{~d} \mathbf{x}+\alpha \int_{\Omega}\left(\varepsilon|\nabla s|^{2}+\frac{1}{4 \varepsilon}(1-s)^{2}\right) \mathrm{d} \mathbf{x} \\
& +\frac{\lambda}{2} \int_{\Omega}|\mathbf{u}-\mathbf{g}|^{2} \mathrm{~d} \mathbf{x}
\end{aligned}
$$

for $\mathbf{u}, \mathbf{g} \in H^{1}\left(\Omega, \mathbb{S}^{m-1}\right), s \in H^{1}(\Omega,[0,1]), 0<\varepsilon, k_{\varepsilon} \ll 1$, and $k_{\varepsilon}=o(\varepsilon)$. Here, $s$ is a phase function approximating $1-\chi_{K}$ by penalisation of phase transitions. Ambrosio and Tortorelli showed $\Gamma$-convergence of $A T_{\varepsilon}(\mathbf{u}, s)$ to $G(\mathbf{u})$ in $L^{2}$ in the scalar $([3,4])$ as well as the $\mathbb{S}^{m-1}$-valued case ([4]) for $\varepsilon \rightarrow 0$.

Bellettini and Coscia [8] carried out a finite element approximation of the Mumford-Shah functional in the scalar case, based on this elliptic approximation. They showed that their approximation $G_{\varepsilon, h}: V^{h}(\Omega) \times V^{h}(\Omega,[0,1]) \rightarrow \overline{\mathbb{R}}$ is $\Gamma$-convergent to $G: H^{1}(\Omega) \times H^{1}(\Omega) \rightarrow \overline{\mathbb{R}}$ provided that the mesh size satisfies $h=o\left(k_{\varepsilon}\right)$, and that $S_{u}$ is piecewise $C^{2}$. Here, $V^{h}(\Omega)$ is the continuous, piecewise affine finite element space. Using the approximation result in [28], Bourdin [10] showed that $S_{u}$ need not be piecewise $C^{2}$; and he proposed an algorithm for actual computations - without providing a proof for its convergence, though. The problem here is that the two variables $u$ and $s$ appear strongly coupled in the energy and in the corresponding gradient flow.

As an alternative to the above phase-field approximation of the Mumford-Shah functional, Braides and Dal Maso [13] proposed a non-local approximation approach, on which Cortesani [22] based a $\Gamma$-convergent, vector-valued finite element approximation.

A different motivation for (1.3) comes from the theory of nematic liquid crystals. In order to overcome mathematical difficulties in showing existence and regularity of energy minimising static configurations in the Oseen-Frank model, Lin [39] adapts Ericksen's energy, which he simplifies to (see [39, equation (3.12)])

$$
\int_{\Omega}\left(\frac{1}{2} s^{2}|\nabla \mathbf{n}|^{2}+|\nabla s|^{2}+W_{0}(s)\right) \mathrm{d} \mathbf{x}
$$

with variable degree of orientation $s \in[-1 / 2,1]$ (in experiments, often $s \geqslant 0$ ), and director $\mathbf{n}$, $|\mathbf{n}|=1$ a.e. The strong similarity of this energy to the functional (1.3) lets us hope that our analysis may be of use for this, too.

The overall goal of the present work is to construct and analyse convergent discretisations for a prototype problem with several non-convexities; namely, we consider a non-convex functional (the Mumford-Shah functional) with a non-convex constraint (the sphere constraint), as an extension 
to existing work on convex functionals (in particular harmonic maps) with non-convex constraints, which have been extensively studied (see e.g. $[1,5,6]$ and references therein). In particular, we deal with discretisations of the sphere constraint, which we account for using a projection and a penalisation strategy. The former turns out to deliver more convincing computational results, while the latter is analytically more satisfactory.

Below, we give a short overview of the two methods for the approximation of (1.1) that we shall present in Sections 3-7 of this paper, where in particular we discuss relevant stability properties of computed approximations, such as

- energy decay property for splitting schemes related to (1.3),

- the validity of a discrete or penalised sphere constraint for approximations of $\mathbf{u}$, and

- non-negativity and upper bounds for approximations of the phase field function $s$.

\subsection{Splitting \& Projection strategy}

The problem of coupled variables is addressed through an iterative splitting strategy, i.e., in every step of the iteration the energy is first minimised with respect to the first variable while keeping the second variable fixed, and then minimised with respect to the second variable while keeping the first one fixed. A special projection idea as proposed by Alouges [1] is used to enforce the sphere constraint. We propose a first-order finite element discretisation, which preserves the sphere constraint exactly at nodal points. The resulting discrete algorithm is simple, results in only linear equations to be solved in every step of the iteration, and every step is energy-decreasing (for acute triangulations). The algorithm converges weakly (up to subsequences) in $H^{1} \times H^{1}$ to a tuple (u, $\left.s\right) \in$ $H^{1}\left(\Omega, \mathbb{S}^{m-1}\right) \times H^{1}(\Omega)$. For $d=2$ we can show that $s$ and iterates $S_{n}$ satisfy $S_{n}, s \in[-1,1]$. However, we have not been able to show that $(\mathbf{u}, s)$ is a stationary point of the Ambrosio-Tortorelli energy for unit vector fields.

\subsection{Penalisation \& Splitting strategy}

This method again uses a splitting strategy, but the sphere constraint is now approximated by penalisation, i.e., we add a Ginzburg-Landau term $\left(4 \delta_{\varepsilon}\right)^{-1} \int_{\Omega}\left(|\mathbf{u}|^{2}-1\right)^{2} \mathrm{~d} \mathbf{x}(0<\delta \ll 1)$ to the energy (1.3). We show that for proper scales of $\delta_{\varepsilon}$ in terms of $\varepsilon$, this does not affect $\Gamma$ convergence. Furthermore, we propose a first-order finite element algorithm based on this splitting and penalisation strategy. The resulting algorithm converges weakly (up to subsequences) in $H^{1} \times H^{1}$ to a tuple $(\mathbf{u}, s) \in H^{1}\left(\Omega, \mathbb{R}^{m}\right) \times H^{1}(\Omega)$, without any mesh constraint. For $d=2$ we can also show that $S_{n}, s \in[-1,1]$. This allows one to get strong convergence (up to subsequences) of iterates $\mathbf{U}_{n}$ in $H^{1}$, which in turn allows passing to the limit and showing that $(\mathbf{u}, s)$ is a stationary point of the Ambrosio-Tortorelli-Ginzburg-Landau energy, and that $s \geqslant 0$. However, we now have to solve a non-linear equation in every iteration.

In Section 6, comparative computational experiments for the "Penalisation \& Splitting" and "Splitting \& Projection" methods are presented, which address in particular

(1) the effect of perturbing the sphere constraint throughout minimisation, as well as proper scalings of regularisation and numerical parameters;

(2) the accuracy of zero sets of $s$ in the course of minimisation; and

(3) comparative numerical studies to relate the CB and RGB models in colour image segmentation. 


\section{Preliminaries}

We often use $c$ and $C$ as generic non-negative constants, capital letters for finite element functions, and boldface for vectors or vector-valued functions. Given $\mathbf{x}, \mathbf{y} \in \mathbb{R}^{d},\langle\mathbf{x}, \mathbf{y}\rangle$ or $\mathbf{x} \cdot \mathbf{y}$ will denote their standard scalar product, and $|\mathbf{x}|$ the Euclidean norm of $\mathbf{x}$. For a measurable set $S,|S|$ or $\mathcal{L}^{d}(S)$ denotes its Lebesgue measure of dimension $d$, and $\mathcal{H}^{d}(S)$ its Hausdorff measure. The $L^{2}$ scalar product and norm will be denoted by $(\cdot, \cdot)$ and $\|\cdot\|$, respectively, and $\mathbb{S}^{m-1}$ will be the unit sphere in $\mathbb{R}^{m}$. For $a, b \in \mathbb{R}$, let $a \wedge b:=\min \{a, b\}$ and $a \vee b:=\max \{a, b\}$. By $A: B$ for $A, B \in \mathbb{R}^{m \times m}$ we denote the dyadic product, i.e., $A: B:=\sum_{i, j=1}^{m} a_{i j} b_{i j}$ for $A=\left(a_{i j}\right), B=\left(b_{i j}\right)$. Let $|A|$ denote the Frobenius norm of $A$, i.e., $|A|^{2}:=\sum_{i, j=1}^{m}\left|a_{i j}\right|^{2}$. For two vectors $\mathbf{a} \in \mathbb{R}^{d}, \mathbf{b} \in \mathbb{R}^{m}$, let $\mathbf{a} \otimes \mathbf{b}:=M$ denote the matrix with entries $m_{i j}:=\mathbf{a}_{i} \mathbf{b}_{j}$.

\section{$2.1 \quad$ Functions of bounded variation and $\Gamma$-convergence}

We summarise some definitions and results on functions of bounded variation and $\Gamma$-convergence. Sources are e.g. [2, 35, 30, 23, 11, 12, 18].

2.1.1 BV, SBV, and GSBV functions. Let $\Omega \subset \mathbb{R}^{d}$ be a bounded open set, $\mathbf{u}: \Omega \rightarrow \mathbb{R}^{m}$ a measurable function, $S:=\mathbb{R}^{m} \cup\{\infty\}$, and $\mathbf{x} \in \Omega$ be fixed. We call $\mathbf{z} \in S$ the approximate limit of

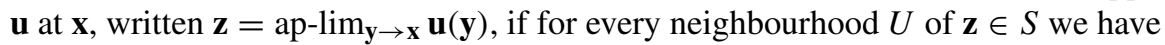

$$
\lim _{\varrho \rightarrow \infty} \frac{1}{\varrho^{n}}|\{\mathbf{y} \in \Omega:|\mathbf{y}-\mathbf{x}|<\varrho, \mathbf{u}(\mathbf{y}) \notin U\}|=0 .
$$

If $\mathbf{z} \in \mathbb{R}^{m}$, we call $\mathbf{x}$ a Lebesgue point of $\mathbf{u}$, and we denote by $S_{\mathbf{u}}$ the complement of the set of Lebesgue points of $\mathbf{u}$ (approximate discontinuity set). Since $\left|S_{\mathbf{u}}\right|$ is known to be zero, $\mathbf{u}=\widetilde{\mathbf{u}}$ a.e. for

$$
\widetilde{\mathbf{u}}(\mathbf{x}):=\underset{\substack{\mathbf{y} \rightarrow \mathbf{x} \\ \mathbf{y} \in \Omega}}{\operatorname{ap}} \lim _{\mathbf{x}} \mathbf{u}(\mathbf{y})
$$

Let $\mathbf{x} \in \Omega \backslash S_{\mathbf{u}}$ be such that $\widetilde{\mathbf{u}}(\mathbf{x}) \neq \infty$. If there exists $L \in \mathbb{R}^{d \times m}$ such that

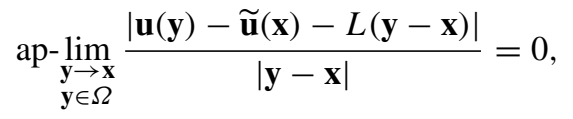

we call $\mathbf{u}$ approximately differentiable at $\mathbf{x}$, and $\nabla \mathbf{u}(\mathbf{x}):=L$ the (uniquely determined) approximate gradient of $\mathbf{u}$ at $\mathbf{x}$. A function $\mathbf{u} \in L^{1}\left(\Omega, \mathbb{R}^{m}\right)$ is called a function of bounded variation in $\Omega$, written $\mathbf{u} \in B V\left(\Omega, \mathbb{R}^{m}\right)$, if its distributional derivative $D \mathbf{u}$ is representable by a measure with finite total variation $|D \mathbf{u}|(\Omega)$, i.e.,

$$
\sum_{\alpha=1}^{m} \int_{\Omega} u^{\alpha} \operatorname{div}(\varphi)^{\alpha} \mathrm{d} \mathbf{x}=-\sum_{\alpha=1}^{m} \sum_{i=1}^{d} \int_{\Omega} \varphi_{i}^{\alpha} \mathrm{d} D_{i} u^{\alpha} \quad \forall \varphi \in C_{c}^{1}\left(\Omega, \mathbb{R}^{m \times d}\right),
$$

with $D \mathbf{u}$ an $\mathbb{R}^{d \times m}$-valued matrix of measures $D_{i} u^{\alpha}$, and $\mathbf{u}=\left(u_{1}, \ldots, u_{m}\right)$. Defining

$$
\|\mathbf{u}\|_{B V\left(\Omega, \mathbb{R}^{m}\right)}:=\|\mathbf{u}\|_{L^{1}\left(\Omega, \mathbb{R}^{m}\right)}+|D \mathbf{u}|(\Omega)
$$

makes $B V\left(\Omega, \mathbb{R}^{m}\right)$ a Banach space. 
If $\left\{\mathbf{u}_{j}\right\} \subset B V\left(\Omega, \mathbb{R}^{m}\right)$ with $\sup _{j}\left\|\mathbf{u}_{j}\right\|_{B V\left(\Omega, \mathbb{R}^{m}\right)}<+\infty$, then there exist a subsequence $\left\{\mathbf{u}_{j_{k}}\right\}$ and a function $\mathbf{u} \in B V\left(\Omega, \mathbb{R}^{m}\right)$ such that $\mathbf{u}_{j_{k}} \rightarrow \mathbf{u}$ in $L^{1}\left(\Omega, \mathbb{R}^{m}\right)$, and $D \mathbf{u}_{j_{k}} \rightarrow D \mathbf{u}$ weakly-* in the sense of measures.

Also, for $\mathbf{u} \in B V\left(\Omega, \mathbb{R}^{m}\right), S_{\mathbf{u}}$ is countably $\mathcal{H}^{d-1}$-rectifiable, i.e.,

$$
S_{\mathbf{u}}=N \cup \bigcup_{i \in \mathbb{N}} K_{i},
$$

where $\mathcal{H}^{d-1}(N)=0$, and each $K_{i}$ is a compact subset of a $C^{1}$ manifold. So, for $\mathcal{H}^{d-1}$-a.e. $\mathbf{y} \in S_{\mathbf{u}}$ we can define the exterior unit normal $\boldsymbol{v}_{\mathbf{u}}$ and outer and inner traces of $\mathbf{u}$ on $S_{\mathbf{u}}$ by

$$
\mathbf{u}^{ \pm}(\mathbf{x}):=\operatorname{ap}-\lim _{\substack{\mathbf{y} \rightarrow \mathbf{x} \\ \mathbf{y} \in \pi^{ \pm}\left(\mathbf{x}, \boldsymbol{v}_{\mathbf{u}}(\mathbf{x})\right)}} \mathbf{u}(\mathbf{y}),
$$

with $\pi^{ \pm}\left(\mathbf{x}, \boldsymbol{v}_{\mathbf{u}}(\mathbf{x})\right):=\left\{\mathbf{y} \in \mathbb{R}^{d}: \pm\left\langle\mathbf{y}-\mathbf{x}, \boldsymbol{v}_{\mathbf{u}}(\mathbf{x})\right\rangle>0\right\}$. A point $\mathbf{x} \in \Omega$ is called a jump point of $\mathbf{u}$, written $\mathbf{x} \in J_{\mathbf{u}}$, if there exists $\boldsymbol{v} \in \mathbb{S}^{d-1}$ such that

$$
\text { ap- } \lim _{\substack{\mathbf{y} \rightarrow \mathbf{x} \\ \mathbf{y} \in \pi^{-}(\mathbf{x}, \boldsymbol{v})}} \mathbf{u}(\mathbf{y}) \neq \text { ap- } \lim _{\substack{\mathbf{y} \rightarrow \mathbf{x} \\ \mathbf{y} \in \pi^{+}(\mathbf{x}, \boldsymbol{v})}} \mathbf{u}(\mathbf{y}) .
$$

It is known that $J_{\mathbf{u}} \subseteq S_{\mathbf{u}}$, and $\mathcal{H}^{d-1}\left(S_{\mathbf{u}} \backslash J_{\mathbf{u}}\right)=0$.

If we decompose $D \mathbf{u}$ into the absolutely continuous part $D^{a} \mathbf{u}$ and the singular part $D^{s} \mathbf{u}$, both with respect to the Lebesgue measure $\mathcal{L}^{d}, D \mathbf{u}=D^{a} \mathbf{u}+D^{s} \mathbf{u}$, then the density of $D^{a} \mathbf{u}$ with respect to $\mathcal{L}^{d}$ coincides with the approximate gradient $\nabla \mathbf{u} \mathcal{L}^{d}$-a.e. The restriction $D^{j} \mathbf{u}$ of $D^{s} \mathbf{u}$ to $S_{\mathbf{u}}$ is called the jump part of Du , and the restriction $D^{c} \mathbf{u}$ of $D^{S} \mathbf{u}$ to $\Omega \backslash S_{\mathbf{u}}$ is called the Cantor part. So,

$$
D \mathbf{u}=D^{a} \mathbf{u}+D^{j} \mathbf{u}+D^{c} \mathbf{u} .
$$

It is known that $D^{j} \mathbf{u}=\left(\mathbf{u}^{+}-\mathbf{u}^{-}\right) \otimes \boldsymbol{v}_{\mathbf{u}} \mathcal{H}^{d-1}\left\lfloor S_{\mathbf{u}}\right.$

A function $\mathbf{u} \in B V\left(\Omega, \mathbb{R}^{m}\right)$ is called a special function of bounded variation in $\Omega$, written $\mathbf{u} \in \operatorname{SBV}\left(\Omega, \mathbb{R}^{m}\right)$, if $D^{c} \mathbf{u}=0$. We call $\mathbf{u} \in B V\left(\Omega, \mathbb{R}^{m}\right)$ a generalised special function of bounded variation, and write $\mathbf{u} \in \operatorname{GSBV}\left(\Omega, \mathbb{R}^{m}\right)$, if $\mathbf{g}(\mathbf{u}) \in \operatorname{SBV}\left(\Omega, \mathbb{R}^{m}\right)$ for every $\mathbf{g} \in C^{1}\left(\mathbb{R}^{m}\right)$ such that $\nabla \mathbf{g}$ has compact support. For $1<p<+\infty$, let

$$
(G) S B V^{p}\left(\Omega, \mathbb{R}^{m}\right):=\left\{\mathbf{u} \in(G) \operatorname{SBV}\left(\Omega, \mathbb{R}^{m}\right): \mathcal{H}^{d-1}\left(J_{\mathbf{u}}\right)<+\infty, \nabla \mathbf{u} \in L^{p}\left(\Omega, \mathbb{R}^{d \times m}\right)\right\} .
$$

We remark that $W^{1,1}\left(\Omega, \mathbb{R}^{m}\right) \subsetneq B V\left(\Omega, \mathbb{R}^{m}\right) ; \mathbf{u} \in \operatorname{SBV}\left(\Omega, R^{m}\right)$ implies $\mathbf{u} \in W^{1,1}\left(\Omega \backslash \overline{S_{\mathbf{u}}}, \mathbb{R}^{m}\right)$; and $\operatorname{SBV}\left(\Omega, \mathbb{R}^{m}\right) \cap L^{\infty}\left(\Omega, \mathbb{R}^{m}\right)=\operatorname{GSBV}\left(\Omega, \mathbb{R}^{m}\right) \cap L^{\infty}\left(\Omega, \mathbb{R}^{m}\right)$.

2.1.2 $\Gamma$-convergence. Let $X$ be a separable Banach space with a topology $\tau$ and let $F_{\varepsilon}: X \rightarrow \overline{\mathbb{R}}$ be a sequence of functionals. We say $F_{\varepsilon} \Gamma$-converges to $F$ in the topology $\tau$, written $F=$ $\Gamma$ - $\lim _{\varepsilon \rightarrow 0} F_{\varepsilon}$, if the following two conditions hold:

(1) For every $x \in X$ and for every sequence $\left\{x_{\varepsilon}\right\} \subset X \tau$-converging to $x \in X$,

$$
F(x) \leqslant \liminf _{\varepsilon \rightarrow 0} F_{\varepsilon}\left(x_{\varepsilon}\right) .
$$


(2) For every $x \in X$ there exists a sequence $\left\{x_{\varepsilon}\right\} \subset X$ (recovery sequence) $\tau$-converging to $x \in X$ such that

$$
F(x) \geqslant \limsup _{\varepsilon \rightarrow 0} F_{\varepsilon}\left(x_{\varepsilon}\right) .
$$

Lemma 2.1 Let $F_{\varepsilon}, F: X \rightarrow \overline{\mathbb{R}}$ with $\Gamma-\lim _{\varepsilon \rightarrow 0} F_{\varepsilon}=F$. Then

(1) $F$ is lower semicontinuous on $X$.

(2) $F+G=\Gamma-\lim \left(F_{\varepsilon}+G\right)$ for all continuous $G: X \rightarrow \mathbb{R}$.

(3) Let $\left\{\mathbf{u}_{\varepsilon}\right\} \subset X$ be such that

$$
\lim _{\varepsilon \rightarrow 0^{+}}\left(F_{\varepsilon}\left(\mathbf{u}_{\varepsilon}\right)-\inf _{X} F_{\varepsilon}\right)=0 .
$$

Then every accumulation point $\mathbf{u}$ of $\left\{\mathbf{u}_{\varepsilon}\right\}$ minimises $F$ over $X$, and

$$
\lim _{\varepsilon \rightarrow 0^{+}} \inf _{X} F_{\varepsilon}=\min _{X} F=F(\mathbf{u}) .
$$

Here are some connections between $\Gamma$-convergence and pointwise convergence:

- If $F_{\varepsilon}$ converges uniformly to $F$, then $F_{\varepsilon} \Gamma$-converges to $F$.

- If $F_{\varepsilon}$ is decreasing and converges pointwise to $F$, then $F_{\varepsilon} \Gamma$-converges to $R F$, the lower semicontinuous envelope of $F$.

\section{Splitting \& Projection algorithm}

Let $\Omega \subset \mathbb{R}^{d}$ be a polyhedral Lipschitz domain, and $\mathcal{T}_{h}$ be a quasi-uniform triangulation of $\Omega$ with node set $\mathcal{N}$ and maximal mesh size $h>0$ (cf. [14]). The space of globally continuous, piecewise affine finite element functions on $\mathcal{T}_{h}$ is denoted by $V_{h}(\Omega) \subseteq H^{1}(\Omega)$. The nodal basis functions are $\left\{\varphi_{\mathbf{z}}: \mathbf{z} \in \mathcal{N}\right\} \subseteq V_{h}(\Omega)$. Let $V_{h}\left(\Omega, \mathbb{R}^{m}\right)$ be the finite element space of $\mathbb{R}^{m}$-valued mappings with basis functions $\left\{\varphi_{\mathbf{z}}^{i}: \mathbf{z} \in \mathcal{N}, 1 \leqslant i \leqslant m\right\}$, with $\varphi_{\mathbf{z}}^{1}:=\left(\varphi_{\mathbf{z}}, 0, \ldots\right)^{T} \in V_{h}\left(\Omega, \mathbb{R}^{m}\right)$, $\varphi_{\mathbf{z}}^{2}:=\left(0, \varphi_{\mathbf{z}}, 0, \ldots\right)^{T} \in V_{h}\left(\Omega, \mathbb{R}^{m}\right)$, and so forth. Let $\mathcal{I}_{h}(\cdot): C^{0}(\bar{\Omega}) \rightarrow V_{h}(\Omega)$ be the Lagrange interpolation operator, and $R_{h}(\cdot): H^{1}(\Omega) \rightarrow V_{h}(\Omega)$ the Ritz projection, defined by

$$
\left(\nabla\left(R_{h}(\varphi)-\varphi\right), \nabla V\right)+\left(R_{h}(\varphi)-\varphi, V\right)=0 \quad \forall V \in V_{h}(\Omega),
$$

and $r_{h}(\cdot): L^{2}(\Omega) \rightarrow V_{h}(\Omega)$ the Clément operator [20] $\left(\mathcal{I}_{h}(\cdot), \mathbf{R}_{h}(\cdot)\right.$, and $\mathbf{r}_{h}(\cdot)$ in the vector-valued case). The latter operator will be needed since it can be applied to discontinuous functions.

LEMma 3.1 The tuple $(\mathbf{u}, s) \in H^{1}\left(\Omega, \mathbb{S}^{m-1}\right) \times H^{1}(\Omega,[0,1])$ is a stationary point of $A T_{\varepsilon}(\cdot, \cdot)$ if and only if

$$
\gamma\left(\left(s^{2}+k_{\varepsilon}\right) \nabla \mathbf{u}, \nabla \varphi\right)=\lambda(\mathbf{g}, \boldsymbol{\varphi})
$$

for all $\varphi \in H^{1}\left(\Omega, \mathbb{R}^{m}\right)$ such that $\varphi(\mathbf{x}) \in \mathrm{T}_{\mathbf{u}(\mathbf{x})} \mathbb{S}^{m-1}$ (the tangent space of $\mathbb{S}^{m-1}$ at $\mathbf{u}(\mathbf{x})$ ), and

$$
2 \alpha \varepsilon(\nabla s, \nabla \varphi)+\left(\left(\gamma|\nabla \mathbf{u}|^{2}+\frac{\alpha}{2 \varepsilon}\right) s, \varphi\right)=\left(\frac{\alpha}{2 \varepsilon}, \varphi\right)
$$

for all $\varphi \in H^{1}(\Omega) \cap L^{\infty}(\Omega)$.

Proof. Note $\mathbf{u} \cdot \boldsymbol{\varphi}=0$ a.e. and derive the first variation of $A T_{\varepsilon}(\cdot, \cdot)$ with respect to $\mathbf{u}$ and $s$, respectively (cf. [49] and [15, Proposition 1.1]). 
The most natural approach to the discrete case would be to work with the original functional $A T_{\varepsilon}(\cdot, \cdot)$. However, it is not clear how to get a uniform $L^{\infty}$ bound on the iterates $S_{n}$ in this setting. We therefore introduce mass lumping into the last term: For $\mathbf{G} \in V_{h}\left(\Omega, \mathbb{R}^{m}\right)$, we define

$$
\begin{aligned}
E_{h}(\mathbf{U}, S):= & \frac{\gamma}{2} \int_{\Omega}\left(S^{2}+k_{\varepsilon}\right)|\nabla \mathbf{U}|^{2} \mathrm{~d} \mathbf{x}+\frac{\lambda}{2} \int_{\Omega}|\mathbf{U}-\mathbf{G}|^{2} \mathrm{~d} \mathbf{x} \\
& +\alpha \int_{\Omega}\left(\varepsilon|\nabla S|^{2}+\frac{1}{4 \varepsilon} \mathcal{I}_{h}\left((1-S)^{2}\right)\right) \mathrm{d} \mathbf{x},
\end{aligned}
$$

and

$$
\widetilde{E}(\mathbf{U}, S):=\frac{\gamma}{2} \int_{\Omega}\left(S^{2}+k_{\varepsilon}\right)|\nabla \mathbf{U}|^{2} \mathrm{~d} \mathbf{x}+\frac{\lambda}{2} \int_{\Omega}|\mathbf{U}-\mathbf{G}|^{2} \mathrm{~d} \mathbf{x},
$$

with $\gamma, \alpha, \varepsilon, k_{\varepsilon}$ fixed and positive, and $\lambda \geqslant 0$. We also assume $d \leqslant 2$, since so far, our arguments for the $L^{\infty}$ bound on the iterates $S_{n}$ fail for higher dimensions (the rest of the analysis works for $d \leqslant 3$ ), but we hope it will be possible to improve these results (and possibly remove lumping altogether).

Another solution would be to use mass lumping in all non-linear terms involving $S$, i.e., to use the functional

$$
\frac{\gamma}{2} \int_{\Omega}\left(\mathcal{I}_{h}\left(S^{2}\right)+k_{\varepsilon}\right)|\nabla \mathbf{U}|^{2} \mathrm{~d} \mathbf{x}+\frac{\lambda}{2} \int_{\Omega}|\mathbf{U}-\mathbf{G}|^{2} \mathrm{~d} \mathbf{x}+\alpha \int_{\Omega}\left(\varepsilon|\nabla S|^{2}+\frac{1}{4 \varepsilon} \mathcal{I}_{h}\left((1-S)^{2}\right)\right) \mathrm{d} \mathbf{x} .
$$

This introduces additional errors, but it still allows one to get the necessary uniform $H^{1}$ bounds on the iterates $\left(\mathbf{U}_{n}, S_{n}\right)$, in addition to the $L^{\infty}$ bound on $S_{n}$, and it does not require $d \leqslant 2$; see [15] for details.

Functions $\mathbf{V} \in V_{h}\left(\Omega, \mathbb{R}^{m}\right)$ which satisfy the pointwise constraint $|\mathbf{V}|=1$ are necessarily constant. So it is more reasonable to work in the space

$$
H_{h}^{1}\left(\mathcal{T}_{h}\right):=\left\{\mathbf{V} \in V_{h}\left(\Omega, \mathbb{R}^{m}\right): \mathbf{V}(\mathbf{z}) \in \mathbb{S}^{m-1} \forall \mathbf{z} \in \mathcal{N}\right\} .
$$

We set

$$
K_{h}^{n}:=\left\{\mathbf{W} \in V_{h}\left(\Omega, \mathbb{R}^{m}\right): \mathbf{W}(\mathbf{z}) \cdot \mathbf{U}_{n}(\mathbf{z})=0 \forall \mathbf{z} \in \mathcal{N}\right\},
$$

where $\mathbf{U}_{n} \in H_{h}^{1}\left(\mathcal{T}_{h}\right)$ will be the iterates of the fully discrete algorithm.

The idea now is to find $\mathbf{U} \in K_{h}^{n}$ minimising $\widetilde{E}(\cdot, S)$ and then project to the sphere. This approach is based on [1] and [5] and replaces the non-linear, non-convex constraint $\mathbf{U} \in H_{h}^{1}\left(\mathcal{T}_{h}\right)$ by the linear one $\mathbf{W}(\mathbf{z}) \cdot \mathbf{U}_{n}(\mathbf{z})=0 \forall \mathbf{z} \in \mathcal{N}$, which in turn ensures that projection to the sphere does not increase the energy.

ALgORIthm 3.2 Let a quasi-uniform triangulation $\mathcal{T}_{h}$ of $\Omega$, starting values $\mathbf{U}_{0}, S_{0}$, and parameters $\varepsilon, k_{\varepsilon}, \varrho>0$ be given. For $n:=0,1, \ldots$

(1) Minimise $\widetilde{E}\left(\mathbf{U}_{n}-\mathbf{W}, S_{n}\right)$ for $\mathbf{W} \in K_{h}^{n}$, i.e. solve

$$
\gamma\left(\left(S_{n}^{2}+k_{\varepsilon}\right) \nabla\left(\mathbf{U}_{n}-\mathbf{W}\right), \nabla \mathbf{V}\right)-\lambda(\mathbf{W}+\mathbf{G}, \mathbf{V})=0,
$$

for all $\mathbf{V} \in K_{h}^{n}$, and call the solution $\mathbf{W}_{n}$.

(2) If $\left\|\mathbf{W}_{n}\right\|_{H^{1}\left(\Omega ; \mathbb{R}^{m}\right)} \leqslant \varrho$ set $\mathbf{U}:=\mathbf{U}_{n}, \mathbf{W}:=\mathbf{W}_{n}, S:=S_{n}$ and stop. 
(3) Set

$$
\mathbf{U}_{n+1}:=\sum_{\mathbf{z} \in \mathcal{N}} \frac{\mathbf{U}_{n}(\mathbf{z})-\mathbf{W}_{n}(\mathbf{z})}{\left|\mathbf{U}_{n}(\mathbf{z})-\mathbf{W}_{n}(\mathbf{z})\right|} \boldsymbol{\varphi}_{\mathbf{z}}
$$

(4) Minimise $E_{h}\left(\mathbf{U}_{n+1}, S\right)$ for all $S \in V_{h}(\Omega)$, i.e. solve

$$
2 \alpha \varepsilon(\nabla S, \nabla W)+\gamma\left(S\left|\nabla \mathbf{U}_{n+1}\right|^{2}, W\right)+\frac{\alpha}{2 \varepsilon}(S-1, W)_{h}=0
$$

for all $W \in V_{h}(\Omega)$, and call the solution $S_{n+1}$.

Here $(\varphi, \psi)_{h}:=\int_{\Omega} \mathcal{I}_{h}(\varphi \psi) \mathrm{d} \mathbf{x}$ for $\varphi, \psi \in C(\bar{\Omega})$.

DEFINITION 3.3 Let $\mathcal{T}_{h}$ be a quasi-uniform triangulation of $\Omega$, and $s \in H^{1}(\Omega)$ be fixed. $\mathcal{T}_{h}$ is said to satisfy the energy decreasing condition $(E D)$ if

$$
E_{h}(\mathbf{W}, s) \leqslant E_{h}(\mathbf{V}, s)
$$

for all $\mathbf{V} \in V_{h}\left(\Omega, \mathbb{R}^{m}\right)$ satisfying $|\mathbf{V}(\mathbf{z})| \geqslant 1$ for $\mathbf{z} \in \mathcal{N}$. Here $\mathbf{W} \in V_{h}\left(\Omega, \mathbb{R}^{m}\right)$ is defined by

$$
\mathbf{W}:=\sum_{\mathbf{z} \in \mathcal{N}} \frac{\mathbf{V}(\mathbf{z})}{|\mathbf{V}(\mathbf{z})|} \varphi_{\mathbf{z}}
$$

As demonstrated in [5, Lemma $3.2 \&$ Remarks 3.3], for $d \leqslant 3$ (ED) holds if every angle in $\mathcal{T}_{h}$ is $\leqslant \pi / 2$ (i.e., if the triangulation is acute).

Lemma 3.4 Let $\mathbf{U} \in V_{h}\left(\Omega, \mathbb{R}^{m}\right)$ be given, and $d \leqslant 2$. If $S \in V_{h}(\Omega)$ minimises $E_{h}(\mathbf{U}, \cdot)$, then we can assume $-1 \leqslant S \leqslant 1$.

Proof. We show that from a minimiser $S \in V_{h}(\Omega)$ that does not satisfy $-1 \leqslant S \leqslant 1$, we can always construct one that does. For $a \in \mathbb{R}$ define $\bar{a}:=-1 \vee a \wedge 1$. Note that for this result it is crucial that we have piecewise affine finite element functions.

Step 1: If $a, b \in \mathbb{R}$, then $(\bar{a}+\bar{b})^{2} \leqslant(a+b)^{2}$ and $(\bar{a}-\bar{b})^{2} \leqslant(a-b)^{2}$. A case differentiation gives:

- $a, b \in[-1,1]$ is trivial.

- $a, b>1$ or $a, b<-1 \Rightarrow(\bar{a}+\bar{b})^{2}=2^{2} \leqslant(a+b)^{2}$.

- $a>1, b<-1 \Rightarrow(\bar{a}+\bar{b})^{2}=0 \leqslant(a+b)^{2}$ and $b>1, a<-1$ is symmetrical.

- $a \notin[-1,1], b \in[-1,1] \Rightarrow 0 \leqslant 1+\operatorname{sign}(a b)|b| \leqslant|a|+\operatorname{sign}(a b)|b|$, $\Rightarrow(\bar{a}+\bar{b})^{2}=(1+\operatorname{sign}(a b)|b|)^{2} \leqslant(|a|+\operatorname{sign}(a b)|b|)^{2}=(a+b)^{2}$, and $b \notin[-1,1], a \in[-1,1]$ is symmetrical.

Therefore $(\bar{a}+\bar{b})^{2} \leqslant(a+b)^{2}$, and $(\bar{a}-\bar{b})^{2} \leqslant(a-b)^{2}$ follows by symmetry.

Step 2: We have $-1 \leqslant S \leqslant 1$. In case $-1 \leqslant S \leqslant 1$ should not be true, we replace $S(\mathbf{x})=$ $\sum_{\mathbf{z} \in \mathcal{N}} S(\mathbf{z}) \varphi_{\mathbf{z}}(\mathbf{x})$ by

$$
\bar{S}(\mathbf{x}):=\sum_{\mathbf{z} \in \mathcal{N}}(-1 \vee S(\mathbf{z}) \wedge 1) \varphi_{\mathbf{z}}(\mathbf{x})=\mathcal{I}_{h}(-1 \vee S \wedge 1),
$$

for which clearly $-1 \leqslant \bar{S} \leqslant 1$. We shall prove $E_{h}(\mathbf{U}, \bar{S}) \leqslant E_{h}(\mathbf{U}, S)$, by showing energy-decrease for every term involving $S$, on every triangle $T \in \mathcal{T}_{h}$. Since $\nabla \mathbf{U}$ is constant on every $T$, the terms we 
have to look at are $\int_{T} S^{2} \mathrm{~d} \mathbf{x}, \int_{T}|\nabla S|^{2} \mathrm{~d} \mathbf{x}$, and $\int_{T} \mathcal{I}_{h}\left((1-S)^{2}\right) \mathrm{d} \mathbf{x}$. Let the values of $S$ at the nodal points of $T$ be $S_{0}, \ldots, S_{d}$, let $\bar{S}_{0}, \ldots, \bar{S}_{d}$ be the corresponding values of $\bar{S}$, let $\varphi_{0}, \ldots, \varphi_{d}$ be the corresponding nodal basis functions, and $\mathbf{x}:=\left(x_{1}, \ldots, x_{d}\right)$. By a simple transformation argument, we can restrict ourselves to the standard simplex, which we shall still call $T$. Then

$$
\left.S(\mathbf{x})\right|_{T}=S_{0}+\sum_{i=1}^{d}\left(S_{i}-S_{0}\right) x_{i} \quad \text { and }\left.\quad \nabla S(\mathbf{x})\right|_{T}=\left(S_{1}-S_{0}, \ldots, S_{d}-S_{0}\right) .
$$

For the first term, a calculation yields

$$
\int_{T} S^{2} \mathrm{~d} \mathbf{x}=\frac{2}{(d+2) !} \sum_{i=0}^{d} S_{i} \sum_{j=i}^{d} S_{j}
$$

If $d=1$, then, by Step 1 ,

$$
\begin{aligned}
\int_{T} \bar{S}^{2} \mathrm{~d} \mathbf{x} & =\frac{1}{3}\left(\bar{S}_{0}^{2}+\bar{S}_{0} \bar{S}_{1}+\bar{S}_{1}^{2}\right)=\frac{1}{6}\left(\left(\bar{S}_{0}+\bar{S}_{1}\right)^{2}+\bar{S}_{0}^{2}+\bar{S}_{1}^{2}\right) \\
& \leqslant \frac{1}{6}\left(\left(S_{0}+S_{1}\right)^{2}+S_{0}^{2}+S_{1}^{2}\right)=\int_{T} S^{2} \mathrm{~d} \mathbf{x} .
\end{aligned}
$$

Similarly, if $d=2$,

$$
\begin{aligned}
\int_{T} \bar{S}^{2} \mathrm{~d} \mathbf{x} & =\frac{1}{12}\left(\bar{S}_{0}^{2}+\bar{S}_{1}^{2}+\bar{S}_{2}^{2}+\bar{S}_{0} \bar{S}_{1}+\bar{S}_{0} \bar{S}_{2}+\bar{S}_{1} \bar{S}_{2}\right) \\
& =\frac{1}{24}\left(\left(\bar{S}_{0}+\bar{S}_{1}\right)^{2}+\left(\bar{S}_{0}+\bar{S}_{2}\right)^{2}+\left(\bar{S}_{1}+\bar{S}_{2}\right)^{2}\right) \\
& \leqslant \frac{1}{24}\left(\left(S_{0}+S_{1}\right)^{2}+\left(S_{0}+S_{2}\right)^{2}+\left(S_{1}+S_{2}\right)^{2}\right)=\int_{T} S^{2} \mathrm{~d} \mathbf{x} .
\end{aligned}
$$

Note that both arguments break down for $d \geqslant 3$; in fact, counter-examples are easy to find (cf. Remark 3.5).

The second term gives, by Step 1 and symmetry,

$$
\begin{aligned}
\int_{T}|\nabla \bar{S}|^{2} \mathrm{~d} \mathbf{x} & =\int_{T}\left(\bar{S}_{1}-\bar{S}_{0}, \ldots, \bar{S}_{d}-\bar{S}_{0}\right)^{2} \mathrm{~d} \mathbf{x}=\frac{1}{d !}\left(\left(\bar{S}_{1}-\bar{S}_{0}\right)^{2}+\cdots+\left(\bar{S}_{d}-\bar{S}_{0}\right)^{2}\right) \\
& \leqslant \frac{1}{d !}\left(\left(S_{1}-S_{0}\right)^{2}+\cdots+\left(S_{d}-S_{0}\right)^{2}\right)=\int_{T}|\nabla S|^{2} \mathrm{~d} \mathbf{x} .
\end{aligned}
$$

As for the last term, again by Step 1,

$$
\int_{T} \mathcal{I}_{h}\left((1-\bar{S})^{2}\right) \mathrm{d} \mathbf{x}=\sum_{i=1}^{d+1}\left(1-\bar{S}_{i}\right)^{2} \int_{T} \varphi_{i} \mathrm{~d} \mathbf{x} \leqslant \sum_{i=1}^{d+1}\left(1-S_{i}\right)^{2} \int_{T} \varphi_{i} \mathrm{~d} \mathbf{x}=\int_{T} \mathcal{I}_{h}\left((1-S)^{2}\right) \mathrm{d} \mathbf{x} .
$$

Remark 3.5 For $d=3$, Step 2 in the above proof is wrong: Let $S_{0}:=S_{1}:=S_{2}:=1$, and $S_{3}:=-3 / 2$. Then, by (3.5),

$$
\int_{T} \bar{S}^{2} \mathrm{~d} \mathbf{x}=\frac{1}{60} \sum_{i=0}^{d} \bar{S}_{i} \sum_{j=i}^{d} \bar{S}_{j}=\frac{1}{15}
$$


while

$$
\int_{T} S^{2} \mathrm{~d} \mathbf{x}=\frac{1}{60} \sum_{i=0}^{d} S_{i} \sum_{j=i}^{d} S_{j}=\frac{1}{16} .
$$

We suspect that there exist dimension-dependent constants $c_{d}$ at which one could crop $|S|$, so that the energy is still decreasing (also replacing $(1-s)^{2}$ by $\left.\left(c_{d}-s\right)^{2}\right)$.

LEMmA 3.6 Let $\mathcal{T}_{h}$ be a quasi-uniform triangulation of $\Omega$ satisfying (ED), $\varrho>0$ fixed, $S_{0} \in$ $V_{h}(\Omega)$, and $\mathbf{U}_{0} \in H_{h}^{1}\left(\mathcal{T}_{h}\right)$. Then Algorithm 3.2 terminates within a finite number of iterations with output $(\mathbf{U}, S) \in H_{h}^{1}\left(\mathcal{T}_{h}\right) \times V_{h}(\Omega,[-1,1])$ and $\mathbf{W} \in V_{h}\left(\Omega, \mathbb{R}^{m}\right)$ such that $\|\nabla \mathbf{W}\| \leqslant \varrho$, and $E_{h}(\mathbf{U}, S) \leqslant E_{h}\left(\mathbf{U}_{0}, S_{0}\right)$.

Proof. We proceed by induction. Suppose that for some $n \geqslant 0$ we have $\left(\mathbf{U}_{n}, S_{n}\right) \in H_{h}^{1}\left(\mathcal{T}_{h}\right) \times$ $V_{h}(\Omega)$. The set $K_{h}^{n}$ is a subspace of $V_{h}\left(\Omega, \mathbb{R}^{m}\right)$. Therefore, by Lax-Milgram, there is a unique $\mathbf{W}_{n} \in K_{h}^{n}$ such that (3.3) holds. Since $\mathbf{W}_{n}(\mathbf{z}) \cdot \mathbf{U}_{n}(\mathbf{z})=0$ and $\left|\mathbf{U}_{n}(\mathbf{z})\right|=1$, we have, for $\mathbf{z} \in \mathcal{N}$,

$$
\left|\mathbf{U}_{n}(\mathbf{z})-\mathbf{W}_{n}(\mathbf{z})\right|^{2}=1+\left|\mathbf{W}_{n}(\mathbf{z})\right|^{2} \geqslant 1 .
$$

Therefore, $\mathbf{U}_{n+1}$ is well-defined and in $H_{h}^{1}\left(\mathcal{T}_{h}\right)$. And since $\mathbf{0} \in K_{h}^{n}$ and $\mathcal{T}_{h}$ satisfies (ED), we get

$$
E_{h}\left(\mathbf{U}_{n+1}, S_{n}\right) \leqslant E_{h}\left(\mathbf{U}_{n}-\mathbf{W}_{n}, S_{n}\right) .
$$

Step 4 of Algorithm 3.2 has a solution $S_{n+1}$ by convexity and coercivity of the functional. So

$$
E_{h}\left(\mathbf{U}_{n+1}, S_{n+1}\right) \leqslant E_{h}\left(\mathbf{U}_{n+1}, S_{n}\right) \leqslant E_{h}\left(\mathbf{U}_{n}-\mathbf{W}_{n}, S_{n}\right) \leqslant E_{h}\left(\mathbf{U}_{n}, S_{n}\right) .
$$

In fact, $E_{h}\left(\mathbf{U}_{n+1}, S_{n+1}\right) \leqslant E_{h}\left(\mathbf{U}_{n+1}, W\right)$ for all $W \in V_{h}(\Omega)$. Therefore, by Lemma 3.4, we can assume $-1 \leqslant S_{n+1} \leqslant 1$. Furthermore,

$$
\begin{aligned}
I:= & 2 \widetilde{E}\left(\mathbf{U}_{n+1}, S_{n+1}\right)-2 \widetilde{E}\left(\mathbf{U}_{n}, S_{n}\right) \\
\leqslant & 2 \widetilde{E}\left(\mathbf{U}_{n}-\mathbf{W}_{n}, S_{n}\right)-2 \widetilde{E}\left(\mathbf{U}_{n}, S_{n}\right) \\
\leqslant & \gamma \int_{\Omega}\left(S_{n}^{2}+k_{\varepsilon}\right)\left(\left|\nabla \mathbf{U}_{n}\right|^{2}+\left|\nabla \mathbf{W}_{n}\right|^{2}-2 \nabla \mathbf{U}_{n}: \nabla \mathbf{W}_{n}\right) \mathrm{d} \mathbf{x} \\
& +\lambda \int_{\Omega}\left(\left|\mathbf{U}_{n}\right|^{2}+\left|\mathbf{W}_{n}\right|^{2}+|\mathbf{G}|^{2}-2 \mathbf{G} \cdot\left(\mathbf{U}_{n}-\mathbf{W}_{n}\right)-2 \mathbf{U}_{n} \cdot \mathbf{W}_{n}\right) \mathrm{d} \mathbf{x} \\
& -\int_{\Omega}\left(\gamma\left(S_{n}^{2}+k_{\varepsilon}\right)\left|\nabla \mathbf{U}_{n}\right|^{2}+\lambda\left(\left|\mathbf{U}_{n}\right|^{2}+|\mathbf{G}|^{2}-2 \mathbf{G} \cdot \mathbf{U}_{n}\right)\right) \mathrm{d} \mathbf{x} \\
= & \int_{\Omega}\left(\gamma\left(S_{n}^{2}+k_{\varepsilon}\right)\left(\left|\nabla \mathbf{W}_{n}\right|^{2}-2 \nabla \mathbf{U}_{n}: \nabla \mathbf{W}_{n}\right)+\lambda\left(\left|\mathbf{W}_{n}\right|^{2}+2 \mathbf{W}_{n} \cdot\left(\mathbf{G}-\mathbf{U}_{n}\right)\right)\right) \mathrm{d} \mathbf{x} .
\end{aligned}
$$

Using equation (3.3) with $\mathbf{V}:=\mathbf{W}_{n}$, we get

$$
I \leqslant-\int_{\Omega}\left(\gamma\left(S_{n}^{2}+k_{\varepsilon}\right)\left|\nabla \mathbf{W}_{n}\right|^{2}+\lambda\left|\mathbf{W}_{n}\right|^{2}\right) \mathrm{d} \mathbf{x}
$$

whence

$$
0 \leqslant \frac{1}{2} \int_{\Omega}\left(\gamma\left(S_{n}^{2}+k_{\varepsilon}\right)\left|\nabla \mathbf{W}_{n}\right|^{2}+\lambda\left|\mathbf{W}_{n}\right|^{2}\right) \mathrm{d} \mathbf{x} \leqslant \widetilde{E}\left(\mathbf{U}_{n}, S_{n}\right)-\widetilde{E}\left(\mathbf{U}_{n+1}, S_{n+1}\right) .
$$


Summing this from 0 to $N$ leads to

$$
\frac{1}{2} \sum_{n=0}^{N} \int_{\Omega}\left(\gamma\left(S_{n}^{2}+k_{\varepsilon}\right)\left|\nabla \mathbf{W}_{n}\right|^{2}+\lambda\left|\mathbf{W}_{n}\right|^{2}\right) \mathrm{d} \mathbf{x} \leqslant \widetilde{E}\left(\mathbf{U}_{0}, S_{0}\right)-\widetilde{E}\left(\mathbf{U}_{N+1}, S_{N+1}\right)<+\infty,
$$

i.e., the series

$$
\frac{1}{2} \sum_{n \geqslant 0} \int_{\Omega}\left(\gamma\left(S_{n}^{2}+k_{\varepsilon}\right)\left|\nabla \mathbf{W}_{n}\right|^{2}+\lambda\left|\mathbf{W}_{n}\right|^{2}\right) \mathrm{d} \mathbf{x}
$$

is convergent. Therefore, $\left\|\mathbf{W}_{n}\right\|_{H^{1}\left(\Omega ; \mathbb{R}^{m}\right)} \leqslant \varrho$ for $n$ large enough.

REMARK 3.7 Let $\left\{\mathcal{T}_{h_{l}}\right\}$ be a sequence of quasi-uniform triangulations satisfying (ED) with maximal mesh size $h_{l} \rightarrow 0$ for $l \rightarrow+\infty, \varrho_{l} \rightarrow 0$ for $l \rightarrow+\infty$, and $E_{h_{l}}\left(\mathbf{U}_{0}, S_{0}\right) \leqslant C_{0}<$ $+\infty$ independently of $h_{l}$. Let $\left\{\mathbf{U}_{l}, S_{l}\right\}$ be the output of Algorithm 3.2 (after termination) from input $\left(\mathbf{U}_{l}^{0}, S_{l}^{0}, \varrho_{l}\right)$. Then the sequence $\left\{\mathbf{U}_{l}, S_{l}\right\}$ converges weakly in $H^{1}\left(\Omega, \mathbb{R}^{m}\right) \times H^{1}(\Omega)$ (up to subsequences, not relabelled) as $l \rightarrow+\infty$ to a point $(\mathbf{u}, s) \in H^{1}\left(\Omega, \mathbb{S}^{m-1}\right) \times H^{1}(\Omega,[-1,1])$, with $A T_{\varepsilon}(\mathbf{u}, s) \leqslant \liminf _{l} A T_{\varepsilon}\left(\mathbf{U}_{l}, S_{l}\right) \leqslant \liminf _{l} A T_{\varepsilon}\left(\mathbf{U}_{l}^{0}, S_{l}^{0}\right)$.

Proof. By assumption and Lemma 3.6, we have

$$
E_{h_{l}}\left(\mathbf{U}_{l}, S_{l}\right) \leqslant E_{h_{l}}\left(\mathbf{U}_{l}^{0}, S_{l}^{0}\right) \leqslant C_{0},
$$

and $-1 \leqslant S_{l} \leqslant 1$. This implies uniform boundedness of $H^{1}$-norms of iterates $\mathbf{U}_{l}$ and $S_{l}$. Hence we can extract a subsequence that converges weakly in $H^{1} \times H^{1}$ to some map $(\mathbf{u}, s)$. Poincaré's inequality (elementwise), $\left|\mathbf{U}_{l}(\mathbf{z})\right|=1$ for all $\mathbf{z} \in \mathcal{N}_{h_{l}}$, and $\left|\mathbf{U}_{l}\right| \leqslant 1$ a.e. imply

$$
\left\|\left|\mathbf{U}_{l}\right|^{2}-1\right\| \leqslant C h_{l}\left\|2 \mathbf{U}_{l}^{T} \nabla \mathbf{U}_{l}\right\| \leqslant C h_{l} .
$$

So $\mathbf{U}_{l} \rightarrow \mathbf{u}$ a.e. leads to $|\mathbf{u}|=1$ a.e.

Since $H^{1}(\Omega)$ is a Hilbert space and $\left\{\varphi \in H^{1}(\Omega): 0 \leqslant \varphi \leqslant 1\right.$ a.e. $\} \subset H^{1}(\Omega)$ is a closed, convex set, it is weakly closed. Therefore, by the weak convergence $S_{l} \rightarrow s$ in $H^{1}$, we get $-1 \leqslant$ $s \leqslant 1$.

Finally, by weak lower semicontinuity of $A T_{\varepsilon}(\cdot, \cdot)$,

$$
\begin{aligned}
A T_{\varepsilon}(\mathbf{u}, s) & \leqslant \liminf _{l} A T_{\varepsilon}\left(\mathbf{U}_{l}, S_{l}\right) \\
& \leqslant \liminf _{l}\left(E_{h_{l}}\left(\mathbf{U}_{l}, S_{l}\right)+c\left\|\mathcal{I}_{h}\left(\left(1-S_{l}\right)^{2}\right)-\left(1-S_{l}\right)^{2}\right\|_{L^{1}(\Omega)}\right) \\
& \leqslant \liminf _{l}\left(E_{h_{l}}\left(\mathbf{U}_{l}^{0}, S_{l}^{0}\right)+c h_{l}\left\|S_{l}\right\|_{L^{2}(\Omega)}\left\|\nabla S_{l}\right\|_{L^{2}(\Omega)}\right) \leqslant \liminf _{l} E_{h_{l}}\left(\mathbf{U}_{l}^{0}, S_{l}^{0}\right) \\
& \leqslant \liminf _{l}\left(A T_{\varepsilon}\left(\mathbf{U}_{l}^{0}, S_{l}^{0}\right)+c h_{l}\left\|S_{l}^{0}\right\|_{L^{2}(\Omega)}\left\|\nabla S_{l}^{0}\right\|_{L^{2}(\Omega)}\right) \leqslant \liminf _{l} A T_{\varepsilon}\left(\mathbf{U}_{l}^{0}, S_{l}^{0}\right) .
\end{aligned}
$$

REMARK 3.8 We have not been able to prove that $(\mathbf{u}, s)$ is a stationary point of $A T_{\varepsilon}(\cdot, \cdot)$. In particular, equation (3.4) in Step 4 of Algorithm 3.2 is

$$
2 \alpha \varepsilon(\nabla S, \nabla W)+\gamma\left(S\left|\nabla \mathbf{U}_{n+1}\right|^{2}, W\right)+\frac{\alpha}{2 \varepsilon}(S-1, W)_{h}=0
$$


for all $W \in V_{h}(\Omega)$. Identifying limits on a term by term basis would require identifying the limit

$$
\lim _{n \rightarrow+\infty}\left(\left|\nabla \mathbf{U}_{n+1}\right|^{2} S_{n}, W\right),
$$

which so far we have to leave as an open problem.

What is missing for this identification of limits is strong convergence of $\nabla \mathbf{U}_{n}$ in $L^{2}$. This is a fundamental shortcoming also observed in $[1,5]$ for the simpler case of harmonic maps to the sphere. In fact, we are not aware of any algorithm, even in the harmonic mapping case, that simultaneously gives strong convergence of $\nabla \mathbf{U}_{n}$ in $L^{2}$ and ensures the sphere constraint exactly.

However, the algorithm converges, decreases the energy, ensures the sphere constraint exactly and delivers very convincing computational results (indeed, it is faster and delivers better results than the alternative algorithm described in Section 6).

\section{4. $\Gamma$-convergence for Penalisation \& Splitting}

In order to resolve the problems with passing to the limit, we now use a penalisation approach instead of projection. This requires adding a term to the Ambrosio-Tortorelli energy, which penalises the sphere constraint. In this section, we show that this addition does not affect $\Gamma$ convergence to the Mumford-Shah functional, if the penalisation term is properly scaled.

Let $\Omega \subset \mathbb{R}^{d}, \gamma, \alpha, \lambda$ be fixed positive constants, $\varepsilon, \delta_{\varepsilon}>0, k_{\varepsilon} \geqslant 0, \mathbf{g} \in L^{\infty}\left(\Omega, \mathbb{S}^{m-1}\right)$, and $G_{\varepsilon}, G: L^{2}\left(\Omega, \mathbb{R}^{m}\right) \times L^{2}(\Omega) \rightarrow[0,+\infty]$ be defined by

$G_{\varepsilon}(\mathbf{u}, s):=\left\{\begin{array}{cc}\frac{\gamma}{2} \int_{\Omega}\left(s^{2}+k_{\varepsilon}\right)|\nabla \mathbf{u}|^{2} \mathrm{~d} \mathbf{x}+\frac{\lambda}{2} \int_{\Omega}|\mathbf{u}-\mathbf{g}|^{2} \mathrm{~d} \mathbf{x}+\alpha \int_{\Omega}\left(\varepsilon|\nabla s|^{2}+\frac{(1-s)^{2}}{4 \varepsilon}\right) \mathrm{d} \mathbf{x} \\ +\frac{1}{4 \delta_{\varepsilon}} \int_{\Omega}\left(|\mathbf{u}|^{2}-1\right)^{2} \mathrm{~d} \mathbf{x} & \text { if } \mathbf{u} \in H^{1}\left(\Omega, \mathbb{R}^{m}\right), s \in H^{1}(\Omega,[0,1]), \\ +\infty, & \text { otherwise, }\end{array}\right.$

and

$G(\mathbf{u}, s)$

$:= \begin{cases}\frac{\gamma}{2} \int_{\Omega}|\nabla \mathbf{u}|^{2} \mathrm{~d} \mathbf{x}+\alpha \mathcal{H}^{d-1}\left(S_{\mathbf{u}}\right)+\frac{\lambda}{2} \int_{\Omega}|\mathbf{u}-\mathbf{g}|^{2} \mathrm{~d} \mathbf{x} & \text { if } \mathbf{u} \in \operatorname{GSBV}\left(\Omega, \mathbb{S}^{m-1}\right) \text { and } s=1 \text { a.e. } \\ +\infty, & \text { otherwise. }\end{cases}$

THEOREM 4.1 If $\Omega \subset \mathbb{R}^{d}$ is open and bounded with Lipschitz boundary, $\delta_{\varepsilon} \rightarrow 0$ as $\varepsilon \rightarrow 0$, $k_{\varepsilon}=o(\varepsilon)$, and $k_{\varepsilon}=o\left(\delta_{\varepsilon}\right)$, then $G_{\varepsilon} \stackrel{\Gamma}{\rightarrow} G$ as $\varepsilon \rightarrow 0$ in $L^{2}\left(\Omega, \mathbb{R}^{m}\right) \times L^{2}(\Omega)$. Moreover, there exists a solution $\left\{\mathbf{u}_{\varepsilon}, s_{\varepsilon}\right\}$ to the minimum problem

$$
m_{\varepsilon}=\inf _{\substack{\mathbf{u} \in H^{1}\left(\Omega, \mathbb{R}^{m}\right), s \in H^{1}(\Omega,[0,1])}} G_{\varepsilon}(\mathbf{u}, s)
$$

with $\left\|\mathbf{u}_{\varepsilon}\right\|_{L^{\infty}} \leqslant C$, and every accumulation point of $\left\{\mathbf{u}_{\varepsilon}\right\}$ is a solution to the minimum problem

$$
m=\inf _{\mathbf{u} \in G S B V\left(\Omega, \mathbb{S}^{m-1}\right)} G(\mathbf{u}, 1)
$$

and $m_{\varepsilon} \rightarrow m$ as $\varepsilon \rightarrow 0^{+}$. 
For the lim inf inequality we can apply the work of Focardi ([32, Lemma 3.3]). For the lim sup inequality we use the same construction as Ambrosio and Tortorelli in [3], so it is enough to verify that the penalisation term we added vanishes for $\varepsilon \rightarrow 0^{+}$. This is explained in more detail below.

Proof. For notational convenience, we first localise the functionals above, denoting by $G_{\varepsilon}(\mathbf{u}, s, A)$ and $G(\mathbf{u}, s, A)$ the same functionals with integration over $A \subseteq \Omega$ instead of $\Omega$, and $\mathcal{H}^{d-1}\left(S_{\mathbf{u}}\right)$ replaced by $\mathcal{H}^{d-1}\left(S_{\mathbf{u}} \cap A\right)$.

Step 1: The liminf inequality. Let $\varepsilon \rightarrow 0^{+}$, and $\left(\mathbf{u}_{\varepsilon}, s_{\varepsilon}\right) \rightarrow(\mathbf{u}, s)$ in $L^{2}\left(\Omega, \mathbb{R}^{m}\right) \times L^{2}(\Omega)$. Up to subsequences, we can suppose that $\left(\mathbf{u}_{\varepsilon}, s_{\varepsilon}\right) \rightarrow(\mathbf{u}, s)$ a.e., and that $\lim _{\varepsilon \rightarrow 0^{+}} G_{\varepsilon}\left(\mathbf{u}_{\varepsilon}, s_{\varepsilon}\right)$ exists and is finite. We can further assume $s=1$ a.e., since otherwise $\int_{\Omega}\left(1-s_{\varepsilon}\right)^{2} \mathrm{~d} \mathbf{x} \nrightarrow 0$, and $G_{\varepsilon}\left(\mathbf{u}_{\varepsilon}, s_{\varepsilon}\right) \rightarrow \infty$. Similarly, we get $|\mathbf{u}|^{2}=1$ a.e.

We now have to show

$$
\liminf _{\varepsilon \rightarrow 0^{+}} G_{\varepsilon}\left(\mathbf{u}_{\varepsilon}, s_{\varepsilon}\right) \geqslant G(\mathbf{u}, s) .
$$

Since it is clear that $\int_{\Omega}\left|\mathbf{u}_{\varepsilon}-\mathbf{g}\right|^{2} \mathrm{~d} \mathbf{x} \rightarrow \int_{\Omega}|\mathbf{u}-\mathbf{g}|^{2} \mathrm{~d} \mathbf{x}$, and that the penalisation term is nonnegative, it is sufficient to prove that $\mathbf{u} \in \operatorname{GSBV}\left(\Omega, \mathbb{R}^{m}\right)$, and

$$
\liminf _{\varepsilon \rightarrow 0^{+}} \int_{\Omega}\left(s_{\varepsilon}^{2}+k_{\varepsilon}\right)\left|\nabla \mathbf{u}_{\varepsilon}\right|^{2} \mathrm{~d} \mathbf{x}+2 \int_{\Omega}\left(\varepsilon\left|\nabla s_{\varepsilon}\right|^{2}+\frac{\left(1-s_{\varepsilon}\right)^{2}}{4 \varepsilon}\right) \mathrm{d} \mathbf{x} \geqslant \int_{\Omega}|\nabla \mathbf{u}|^{2} \mathrm{~d} \mathbf{x}+2 \mathcal{H}^{d-1}\left(S_{\mathbf{u}}\right) .
$$

This was shown for a more general situation in [32, Lemma 3.3] (see also [33]).

Step 2: The limsup inequality. It suffices to consider the case $\mathbf{u} \in \operatorname{SBV}\left(\Omega, \mathbb{R}^{m}\right) \cap L^{\infty}\left(\Omega, \mathbb{R}^{m}\right)$. We can also assume $\nabla \mathbf{u} \in L^{2}\left(\Omega, \mathbb{R}^{d \times m}\right),|\mathbf{u}|^{2}=1$ a.e., and (see [33, Theorem 2.7.14]) that $S_{\mathbf{u}}$ is essentially closed in $\Omega$, i.e., $\mathcal{H}^{d-1}\left(\Omega \cap\left(\overline{S_{\mathbf{u}}} \backslash S_{\mathbf{u}}\right)\right)=0$. Setting $d(\mathbf{x}):=\operatorname{dist}\left(\mathbf{x}, S_{\mathbf{u}}\right)$, we define the Minkowski content of $S_{\mathbf{u}}$ by

$$
\mathcal{M}^{d-1}\left(S_{\mathbf{u}}\right):=\lim _{\delta \rightarrow 0^{+}} \mathcal{M}_{\delta}^{d-1}\left(S_{\mathbf{u}}\right):=\lim _{\delta \rightarrow 0^{+}} \frac{|\{\mathbf{x} \in \Omega: d(\mathbf{x})<\delta\}|}{2 \delta} .
$$

It is well-known that for $S_{\mathbf{u}}$ essentially closed,

$$
\lim _{\delta \rightarrow 0^{+}} \mathcal{M}_{\delta}^{d-1}\left(S_{\mathbf{u}}\right)=\mathcal{H}^{d-1}\left(S_{\mathbf{u}}\right)
$$

(see [31, Theorem 3.2.39]). So, there exists a sequence $w_{\varepsilon} \rightarrow 0^{+}$such that

$$
|\{\mathbf{x} \in \Omega: d(\mathbf{x})<\delta\}| \leqslant 2 \delta\left(\mathcal{H}^{d-1}\left(S_{\mathbf{u}}\right)+w_{\varepsilon}\right)
$$

for every $\delta \geqslant 0$ small enough.

Given such functions $\mathbf{u}$, and $s=1$ a.e., we have to construct $\left\{\mathbf{u}_{\varepsilon}, s_{\varepsilon}\right\}$ that converge in $L^{2}\left(\Omega, \mathbb{R}^{m}\right) \times L^{2}(\Omega)$ to $(\mathbf{u}, s)$ and such that

$$
\limsup _{\varepsilon \rightarrow 0^{+}} G_{\varepsilon}\left(\mathbf{u}_{\varepsilon}, s_{\varepsilon}\right) \leqslant G(u, s)
$$

for any positive sequence $\varepsilon$ converging to zero.

It is natural to require $s_{\varepsilon} \equiv 0$ in some $\varepsilon$-dependent neighbourhood of $S_{\mathbf{u}}, s_{\varepsilon}$ converging to 1 everywhere outside a larger neighbourhood of $S_{\mathbf{u}}$, and smooth in between, as well as $\mathbf{u}_{\varepsilon} \equiv \mathbf{u}$ everywhere outside some neighbourhood of $S_{\mathbf{u}}$. 
To this end, we use the same construction as in the paper [3] by Ambrosio and Tortorelli: Choose a positive sequence $b_{\varepsilon}$ such that $b_{\varepsilon}=o(\varepsilon), b_{\varepsilon}=o\left(\delta_{\varepsilon}\right)$, and $k_{\varepsilon}=o\left(b_{\varepsilon}\right)$. For any $b>0$, set $S_{b}:=\{\mathbf{x} \in \Omega: d(\mathbf{x})<b\}$. Thanks to (4.2), $\left|S_{b}\right|=O(b)$. For $t \geqslant b_{\varepsilon}$, let

$$
\sigma_{\varepsilon}(t):=1-\exp \left(-\frac{t-b_{\varepsilon}}{2 \varepsilon}\right), \quad \text { so that } \quad \sigma_{\varepsilon}^{\prime}(t)=\frac{1}{2 \varepsilon} \exp \left(-\frac{t-b_{\varepsilon}}{2 \varepsilon}\right) .
$$

We now set (cf. Figure 1)

$$
s_{\varepsilon}(\mathbf{x}):= \begin{cases}0 & \text { if } \mathbf{x} \in S_{b_{\varepsilon}}, \\ \sigma_{\varepsilon}(d(\mathbf{x})) & \text { if } \mathbf{x} \in S_{b_{\varepsilon}+2 \varepsilon \ln (1 / \varepsilon) \backslash S_{b_{\varepsilon}},}, \\ 1-\varepsilon & \text { if } \mathbf{x} \in \Omega \backslash S_{b_{\varepsilon}+2 \varepsilon} \ln (1 / \varepsilon),\end{cases}
$$

and

$$
\mathbf{u}_{\varepsilon}(\mathbf{x}):=\mathbf{u}(\mathbf{x}) \min \left\{d(\mathbf{x}) / b_{\varepsilon}, 1\right\} .
$$

Note that $0<2 \varepsilon \ln (1 / \varepsilon) \rightarrow 0^{+}$, and $\varepsilon=o(2 \varepsilon \ln (1 / \varepsilon))$.

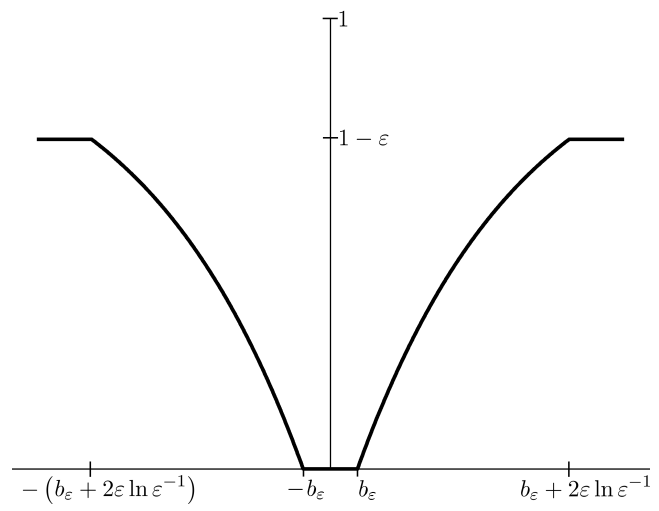

FIG. 1. Sketch of $s_{\mathcal{E}}(\mathbf{x})$ in the case $S_{\mathbf{u}}=\{0\}$, and $d=1$.

By construction, $\left(\mathbf{u}_{\varepsilon}, s_{\varepsilon}\right) \rightarrow(\mathbf{u}, 1)$ in $L^{2}\left(\Omega, \mathbb{R}^{m}\right) \times L^{2}(\Omega)$ as $\varepsilon \rightarrow 0^{+}$.

Therefore, the term penalising the sphere constraint satisfies

$$
\frac{1}{4 \delta_{\varepsilon}} \int_{\Omega}\left(\left|\mathbf{u}_{\varepsilon}\right|^{2}-1\right)^{2} \mathrm{~d} \mathbf{x} \leqslant c \frac{\left|S_{b_{\varepsilon}}\right|}{\delta_{\varepsilon}} \leqslant c \frac{b_{\varepsilon}}{\delta_{\varepsilon}} \rightarrow 0 .
$$

So this term does not contribute to the lim sup. This calculation motivates why we cannot expect good experimental results for $\delta_{\varepsilon}$ too small (compared to $b_{\varepsilon}$, which in turn is between $\varepsilon$ and $k_{\varepsilon}$ ); i.e., we have to sacrifice something in terms of the sphere constraint (cf. our experiments in Section 6.2).

The other terms are just like those in the original paper [3].

Step 3: Convergence of minimisers. The functional $G_{\varepsilon}$ is coercive and lower semicontinuous in $L^{2}$. So for every $\varepsilon>0$ there exists a minimising pair $\left(\mathbf{u}_{\varepsilon}, s_{\varepsilon}\right)$ of $G_{\varepsilon}$. By a simple truncation argument, $\left\|\mathbf{u}_{\varepsilon}\right\|_{L^{\infty}} \leqslant C$. As above, we can assume that $\left(\mathbf{u}_{\varepsilon}, s_{\varepsilon}\right) \in \operatorname{SBV}\left(\Omega, \mathbb{R}^{m}\right) \times S B V(\Omega) \cap L^{\infty}\left(\Omega, \mathbb{R}^{m}\right) \times$ $L^{\infty}(\Omega)$. By the SBV Closure and Compactness Theorems [2, Theorems 4.7 and 4.8], there exists a subsequence $\left\{\mathbf{u}_{\varepsilon_{j}}, s_{\varepsilon_{j}}\right\}$ converging to some $(\mathbf{u}, 1)$ in $L^{2}\left(\Omega, \mathbb{R}^{m}\right) \times L^{2}(\Omega)$, with $\mathbf{u} \in S B V\left(\Omega, \mathbb{R}^{m}\right)$. Thus, the stability of minimising sequences under $\Gamma$-convergence (Lemma 2.1(3)) concludes the proof. 


\section{Penalisation \& Splitting algorithm}

Let $\Omega \subset \mathbb{R}^{d}$ be a polyhedral Lipschitz domain, and let $\mathbf{g}: \Omega \rightarrow \mathbb{S}^{m-1}$ be the chromaticity component of a given image. For $\mathbf{u}, \mathbf{g} \in H^{1}\left(\Omega, \mathbb{R}^{m}\right), s \in H^{1}(\Omega,[0,1])$, and $0<\varepsilon, k_{\varepsilon}, \delta_{\varepsilon} \ll 1$, we want to minimise the following vector-valued Ambrosio-Tortorelli-Ginzburg-Landau energy using a splitting strategy:

$$
\begin{aligned}
G_{\varepsilon}(\mathbf{u}, s)= & \frac{\gamma}{2} \int_{\Omega}\left(s^{2}+k_{\varepsilon}\right)|\nabla \mathbf{u}|^{2} \mathrm{~d} \mathbf{x}+\frac{\lambda}{2} \int_{\Omega}|\mathbf{u}-\mathbf{g}|^{2} \mathrm{~d} \mathbf{x} \\
& +\alpha \int_{\Omega}\left(\varepsilon|\nabla s|^{2}+\frac{1}{4 \varepsilon}(1-s)^{2}\right) \mathrm{d} \mathbf{x}+\frac{1}{4 \delta_{\varepsilon}} \int_{\Omega}\left(|\mathbf{u}|^{2}-1\right)^{2} \mathrm{~d} \mathbf{x} .
\end{aligned}
$$

In this section, we shall always assume that $\gamma, \alpha, \varepsilon, k_{\varepsilon}, \delta_{\varepsilon}$ are fixed and positive, $\lambda \geqslant 0$, and $d \leqslant 2$ (the last assumption is again only used to show that the iterates $S_{n}$ are in $[-1,1]$, and that their weak limit $s$ is in $[0,1])$.

Definition 5.1 A tuple $(\mathbf{u}, s) \in H^{1}\left(\Omega, \mathbb{R}^{m}\right) \times H^{1}(\Omega,[0,1])$ is called a weak solution to the problem $\inf G_{\varepsilon}$ if

$$
\gamma\left(\left(s^{2}+k_{\varepsilon}\right) \nabla \mathbf{u}, \nabla \boldsymbol{\varphi}\right)+\lambda(\mathbf{u}-\mathbf{g}, \boldsymbol{\varphi})+\frac{1}{\delta_{\varepsilon}}\left(\left(|\mathbf{u}|^{2}-1\right) \mathbf{u}, \boldsymbol{\varphi}\right)=0
$$

for all $\varphi \in H^{1}\left(\Omega, \mathbb{R}^{m}\right)$, and

$$
2 \alpha \varepsilon(\nabla s, \nabla \varphi)+\gamma\left(|\nabla \mathbf{u}|^{2} s, \varphi\right)+\frac{\alpha}{2 \varepsilon}(s-1, \varphi)=0
$$

for all $\varphi \in H^{1}(\Omega) \cap L^{\infty}(\Omega)$.

We use the same finite element setting as in Section 3, in particular, we shall always assume that the triangulation $\mathcal{T}_{h}$ is quasi-uniform. For $\mathbf{U}, \mathbf{G} \in V_{h}\left(\Omega, \mathbb{R}^{m}\right)$ and $S \in V_{h}(\Omega,[-1,1])$, let

$$
\begin{aligned}
G_{\varepsilon, h}(\mathbf{U}, S)= & \frac{\gamma}{2} \int_{\Omega}\left(S^{2}+k_{\varepsilon}\right)|\nabla \mathbf{U}|^{2} \mathrm{~d} \mathbf{x}+\frac{\lambda}{2} \int_{\Omega}|\mathbf{U}-\mathbf{G}|^{2} \mathrm{~d} \mathbf{x} \\
& +\alpha \int_{\Omega}\left(\varepsilon|\nabla S|^{2}+\frac{1}{4 \varepsilon} \mathcal{I}_{h}\left((1-S)^{2}\right)\right) \mathrm{d} \mathbf{x}+\frac{1}{4 \delta_{\varepsilon}} \int_{\Omega}\left(|\mathbf{U}|^{2}-1\right)^{2} \mathrm{~d} \mathbf{x} .
\end{aligned}
$$

In the algorithm below we use $\mathbf{G}:=\mathbf{r}_{h}(\mathbf{g}) \in V_{h}\left(\Omega, \mathbb{R}^{m}\right)$, i.e., the Clément interpolation of $\mathbf{g}$. This allows the use of non-smooth $\mathbf{g}$. If $\mathbf{g} \in C^{0}\left(\Omega, \mathbb{R}^{m}\right)$, the Lagrange interpolation would do as well.

Algorithm 5.2 Let $\mathbf{U}_{0}, \mathbf{G} \in V_{h}\left(\Omega, \mathbb{R}^{m}\right)$ and $S_{0} \in V_{h}(\Omega)$ be given. For $n=1,2, \ldots$ until convergence do

(1) Compute $\mathbf{U}_{n} \in V_{h}\left(\Omega, \mathbb{R}^{m}\right)$ such that

$$
\gamma\left(\left(S_{n-1}^{2}+k_{\varepsilon}\right) \nabla \mathbf{U}_{n}, \nabla \mathbf{W}\right)+\lambda\left(\mathbf{U}_{n}-\mathbf{G}, \mathbf{W}\right)+\frac{1}{\delta_{\varepsilon}}\left(\left(\left|\mathbf{U}_{n}\right|^{2}-1\right) \mathbf{U}_{n}, \mathbf{W}\right)=0
$$

for all $\mathbf{W} \in V_{h}\left(\Omega, \mathbb{R}^{m}\right)$. 
(2) Compute $S_{n} \in V_{h}(\Omega)$ such that

$$
2 \alpha \varepsilon\left(\nabla S_{n}, \nabla W\right)+\gamma\left(S_{n}\left|\nabla \mathbf{U}_{n}\right|^{2}, W\right)+\frac{\alpha}{2 \varepsilon}\left(S_{n}-1, W\right)_{h}=0
$$

for all $W \in V_{h}(\Omega)$.

The following existence and uniqueness result follows by standard coercivity and convexity arguments for $G_{\varepsilon, h}$ (see e.g. [34, Section 8.4]). The fact $-1 \leqslant S \leqslant 1$ follows from Lemma 3.4.

Proposition 5.3 There exists a function $\mathbf{U} \in V_{h}\left(\Omega, \mathbb{R}^{m}\right)$ such that (5.5) holds for all $\mathbf{W} \in$ $V_{h}\left(\Omega, \mathbb{R}^{m}\right)$, and there is a unique function $S \in V_{h}(\Omega,[-1,1])$ such that (5.6) holds for all $W \in$ $V_{h}(\Omega)$.

LEMma 5.4 Algorithm 5.2 decreases $G_{\varepsilon, h}$ with respect to $n \in \mathbb{N}$.

Proof. For any $n \in \mathbb{N}$ fixed, Algorithm 5.2 ensures that

$$
G_{\varepsilon, h}\left(\mathbf{U}_{n+1}, S_{n+1}\right) \leqslant G_{\varepsilon, h}\left(\mathbf{U}_{n+1}, S_{n}\right) \leqslant G_{\varepsilon, h}\left(\mathbf{U}_{n}, S_{n}\right) .
$$

The main convergence properties of the iterates from Algorithm 5.2 are given in the following

THEOREM 5.5 Let $\left\{\mathcal{T}_{h_{l}}\right\}$ be a sequence of quasi-uniform triangulations with maximal mesh size $h_{l} \rightarrow 0$ for $l \rightarrow+\infty$, and $G_{\varepsilon, h_{l}}\left(\mathbf{U}_{0}^{l}, S_{0}^{l}\right) \leqslant C_{0}<+\infty$ independently of $h_{l}$. Then the sequences $\left\{\mathbf{U}_{m}^{l}, S_{m}^{l}\right\}_{m, l}$ constructed by Algorithm 5.2 from inputs $\left(\mathbf{U}_{0}^{l}, S_{0}^{l}\right)$ have a (diagonal) subsequence, called $\left\{\mathbf{U}_{n}, S_{n}\right\}_{n}$, such that $\mathbf{U}_{n}$ converges strongly in $H^{1}\left(\Omega, \mathbb{R}^{m}\right)$, and $S_{n}$ converges weakly in $H^{1}(\Omega)$ to some $(\mathbf{u}, s) \in H^{1}\left(\Omega, \mathbb{R}^{m}\right) \times H^{1}(\Omega,[0,1])$, which is a weak solution as in Definition 5.1.

To identify limits in the proof of Theorem 5.5, it will be crucial to prove strong $L^{2}$ convergence of $\nabla \mathbf{U}_{n}$ to $\nabla \mathbf{u}$, for which we use a strategy from [15, Proof of Theorem 4.2], where the authors show convergence of two adaptive, stationary finite element approximations for the minimisation of the unconstrained Ambrosio-Tortorelli energy: In Step 2 we show that $\mathbf{u}$ satisfies (5.2), then we use (5.2) and (5.5) and dominated convergence (cf. Lemma 5.6, also derived from [15]) to show strong $L^{2}$ convergence of $\nabla \mathbf{U}_{n}$ to $\nabla \mathbf{u}$ in Step 3, and finally we use this to show that $s$ satisfies (5.3) in Step 4.

LEMmA 5.6 Let $p_{n}, p \in H^{1}(\Omega) \cap L^{\infty}(\Omega)$ such that $\left\|p_{n}\right\|_{L^{\infty}(\Omega)},\|p\|_{L^{\infty}(\Omega)} \leqslant C<+\infty$ a.e., independently of $n$, and $p_{n} \rightarrow p$ in $L^{2}(\Omega)$. Then

$$
\lim _{n}\left(\left|p_{n}-p\right|,|\nabla \varphi|^{2}\right)=0 \quad \forall \boldsymbol{\varphi} \in H^{1}\left(\Omega, \mathbb{R}^{m}\right) .
$$

Proof. See [15, Proof of Theorem 4.2].

Proof of Theorem 5.5.

Step 1: For $m, l \rightarrow \infty$, there is a subsequence $\left\{\mathbf{U}_{n}, S_{n}\right\}$, converging weakly in $H^{1}\left(\Omega, \mathbb{R}^{m}\right) \times$ $H^{1}(\Omega)$ to some $(\mathbf{u}, s) \in H^{1}\left(\Omega, \mathbb{R}^{m}\right) \times H^{1}(\Omega,[-1,1])$. For every $m, l \in \mathbb{N}$, Proposition 5.3 gives existence of $\left(\mathbf{U}_{m}^{l}, S_{m}^{l}\right)$ and ensures that $-1 \leqslant S_{m}^{l} \leqslant 1$ a.e. By Lemma 5.4 and by assumption,

$$
G_{\varepsilon, h_{l}}\left(\mathbf{U}_{m}^{l}, S_{m}^{l}\right) \leqslant G_{\varepsilon, h_{l}}\left(\mathbf{U}_{0}^{l}, S_{0}^{l}\right) \leqslant C_{0},
$$

independently of $l, m$. In particular, $G_{\varepsilon, h_{n}}\left(\mathbf{U}_{n}^{n}, S_{n}^{n}\right) \leqslant C_{0}$. So, by the definition of $G_{\varepsilon, h_{n}}$, the $H^{1}$ norms of $\mathbf{U}_{n}^{n}$ and $S_{n}^{n}$ are bounded independently of $n$. Therefore, since $H^{1}$ is a Hilbert space, 
there exist subsequences, called $\left\{\mathbf{U}_{n}\right\}$ and $\left\{S_{n}\right\}$, which converge weakly in $H^{1}$ to some $(\mathbf{u}, s) \in$ $H^{1}\left(\Omega, \mathbb{R}^{m}\right) \times H^{1}(\Omega)$.

Finally, since $H^{1}(\Omega)$ is a Hilbert space and $\left\{\varphi \in H^{1}(\Omega):-1 \leqslant \varphi \leqslant 1\right.$ a.e $\} \subset H^{1}(\Omega)$ is a closed, convex set, it is weakly closed. Therefore, by the weak convergence $S_{n} \rightarrow s$ in $H^{1}$, we get $-1 \leqslant s \leqslant 1$.

Below, we shall use the abbreviation $h$ for $h_{n}$.

Step 2: u solves equation (5.2). Let $\varphi \in C^{\infty}\left(\Omega, \mathbb{R}^{m}\right)$ be fixed, $n \in \mathbb{N}$, and $h>0$. Consider

$$
\gamma\left(\left(s^{2}+k_{\varepsilon}\right) \nabla \mathbf{u}, \nabla \varphi\right)+\lambda(\mathbf{u}-\mathbf{g}, \boldsymbol{\varphi})+\frac{1}{\delta_{\varepsilon}}\left(\left(|\mathbf{u}|^{2}-1\right) \mathbf{u}, \boldsymbol{\varphi}\right)=: \gamma T_{1}+\lambda T_{2}+\frac{1}{\delta_{\varepsilon}} T_{3} .
$$

Since $H^{1}$ is compactly embedded in $L^{p}$ for $p<6$, as long as the space dimension $d \leqslant 3$, we have $\mathbf{U}_{n} \rightarrow \mathbf{u}$ in $L^{p}\left(\Omega, \mathbb{R}^{m}\right)$ for $p<6$.

We compute

$$
\begin{aligned}
T_{1}= & \left(\left(S_{n-1}^{2}+k_{\varepsilon}\right) \nabla \mathbf{U}_{n}, \nabla \mathcal{I}_{h}(\boldsymbol{\varphi})\right)+\left(\left(s^{2}-S_{n-1}^{2}\right) \nabla \mathbf{U}_{n}, \nabla \boldsymbol{\varphi}\right) \\
& +\left(\left(s^{2}+k_{\varepsilon}\right) \nabla\left(\mathbf{u}-\mathbf{U}_{n}\right), \nabla \boldsymbol{\varphi}\right)+\left(\left(S_{n-1}^{2}+k_{\varepsilon}\right) \nabla \mathbf{U}_{n}, \nabla\left(\boldsymbol{\varphi}-\mathcal{I}_{h}(\boldsymbol{\varphi})\right)\right) \\
= & : T_{11}^{n}+T_{12}^{n}+T_{13}^{n}+T_{14}^{n} .
\end{aligned}
$$

Note that $\left\|\boldsymbol{\varphi}-\mathcal{I}_{h}(\boldsymbol{\varphi})\right\|_{H^{r}\left(\Omega, \mathbb{R}^{m}\right)} \leqslant c h^{2-r}\left\|\nabla^{2} \boldsymbol{\varphi}\right\|_{L^{2}\left(\Omega, \mathbb{R}^{m}\right)}$ for $0 \leqslant r \leqslant 2$.

Since $-1 \leqslant S_{n-1}, s \leqslant 1$, we have $\left|S_{n-1}^{2}-s^{2}\right| \leqslant C\left|S_{n-1}-s\right| \leqslant C\left|S_{n-1}-s\right|^{1 / 2}$, whence, by Lemma 5.6,

$$
T_{12}^{n}=\left(\left(s^{2}-S_{n-1}^{2}\right) \nabla \mathbf{U}_{n}, \nabla \varphi\right) \leqslant C\left(\left|s-S_{n-1}\right|,|\nabla \varphi|^{2}\right)^{1 / 2}\left\|\nabla \mathbf{U}_{n}\right\|_{L^{2}\left(\Omega, \mathbb{R}^{m \times d}\right)}^{\stackrel{h \rightarrow 0}{n \rightarrow+\infty}} 0 .
$$

Since $s \leqslant 1$, we know that $\left(s^{2}+k_{\varepsilon}\right) \nabla \varphi \in L^{2}\left(\Omega, \mathbb{R}^{d \times m}\right)$, so $T_{13}^{n}=\left(\nabla\left(\mathbf{u}-\mathbf{U}_{n}\right),\left(s^{2}+k_{\varepsilon}\right) \nabla \varphi\right) \rightarrow 0$, by weak convergence. And since $\left\|\boldsymbol{\varphi}-\mathcal{I}_{h}(\boldsymbol{\varphi})\right\|_{H^{1}\left(\Omega, \mathbb{R}^{m}\right)} \rightarrow 0$, in view of the bounds established in Step 1 , the terms $T_{14}^{n}, T_{2}, T_{3}$ all clearly vanish.

Putting all of the above together, we have for $n \in \mathbb{N}$ and $h>0$ fixed,

$$
\gamma\left(\left(s^{2}+k_{\varepsilon}\right) \nabla \mathbf{u}, \nabla \boldsymbol{\varphi}\right)+\lambda(\mathbf{u}-\mathbf{g}, \boldsymbol{\varphi})+\frac{1}{\delta_{\varepsilon}}\left(\left(|\mathbf{u}|^{2}-1\right) \mathbf{u}, \boldsymbol{\varphi}\right)=: \gamma T_{13}^{n}+\lambda T_{21}^{n}+\frac{1}{\delta_{\varepsilon}} T_{31}^{n}+T^{n},
$$

where $\gamma T_{13}^{n}+\lambda T_{21}^{n}+1 / \delta_{\varepsilon} T_{31}^{n}=0$ by construction. Now, letting $n \rightarrow+\infty$ and $h \rightarrow 0$, we have $T^{n} \rightarrow 0$, as shown above. And by a density argument, the above is true for general $\varphi \in H^{1}\left(\Omega, \mathbb{R}^{m}\right)$. Step 3: $\nabla \mathbf{U}_{n} \rightarrow \nabla \mathbf{u}$ strongly in $L^{2}\left(\Omega, \mathbb{R}^{m \times d}\right)$ as $n \rightarrow+\infty$ and $h \rightarrow 0$. Let $n \in \mathbb{N}$ and $h>0$. Then

$$
\begin{aligned}
\gamma k_{\varepsilon}\left\|\nabla\left(\mathbf{u}-\mathbf{U}_{n}\right)\right\|_{L^{2}}^{2} \leqslant & \gamma\left(\left(S_{n-1}^{2}+k_{\varepsilon}\right) \nabla \mathbf{u}, \nabla\left(\mathbf{R}_{h}(\mathbf{u})-\mathbf{U}_{n}\right)\right) \\
& -\gamma\left(\left(S_{n-1}^{2}+k_{\varepsilon}\right) \nabla \mathbf{U}_{n}, \nabla\left(\mathbf{R}_{h}(\mathbf{u})-\mathbf{U}_{n}\right)\right) \\
& -\lambda\left(\mathbf{U}_{n}-\mathbf{G}, \mathbf{R}_{h}(\mathbf{u})-\mathbf{U}_{n}\right)-\frac{1}{\delta_{\varepsilon}}\left(\left(\left|\mathbf{U}_{n}\right|^{2}-1\right) \mathbf{U}_{n}, \mathbf{R}_{h}(\mathbf{u})-\mathbf{U}_{n}\right) \\
& +\lambda\left(\mathbf{U}_{n}-\mathbf{G}, \mathbf{R}_{h}(\mathbf{u})-\mathbf{U}_{n}\right)+\frac{1}{\delta_{\varepsilon}}\left(\left(\left|\mathbf{U}_{n}\right|^{2}-1\right) \mathbf{U}_{n}, \mathbf{R}_{h}(\mathbf{u})-\mathbf{U}_{n}\right) \\
& +\gamma\left(\left(S_{n-1}^{2}+k_{\varepsilon}\right) \nabla\left(\mathbf{u}-\mathbf{U}_{n}\right), \nabla\left(\mathbf{u}-\mathbf{R}_{h}(\mathbf{u})\right)\right) \\
= & T_{1}^{n}+\cdots+T_{7}^{n} .
\end{aligned}
$$


By construction (equation (5.5) with $\mathbf{W}:=\mathbf{R}_{h}(\mathbf{u})-\mathbf{U}_{n}$ ), the expression $T_{2}^{n}+T_{3}^{n}+T_{4}^{n}$ is zero. Next,

$$
\begin{aligned}
T_{1}^{n}= & \gamma\left(\left(S_{n-1}^{2}-s^{2}\right) \nabla \mathbf{u}, \nabla\left(\mathbf{R}_{h}(\mathbf{u})-\mathbf{U}_{n}\right)\right)+\gamma\left(\left(s^{2}+k_{\varepsilon}\right) \nabla \mathbf{u}, \nabla\left(\mathbf{R}_{h}(\mathbf{u})-\mathbf{U}_{n}\right)\right) \\
& +\lambda\left(\mathbf{u}-\mathbf{g}, \mathbf{R}_{h}(\mathbf{u})-\mathbf{U}_{n}\right)+\frac{1}{\delta_{\varepsilon}}\left(\left(|\mathbf{u}|^{2}-1\right) \mathbf{u}, \mathbf{R}_{h}(\mathbf{u})-\mathbf{U}_{n}\right) \\
& -\lambda\left(\mathbf{u}-\mathbf{g}, \mathbf{R}_{h}(\mathbf{u})-\mathbf{U}_{n}\right)-\frac{1}{\delta_{\varepsilon}}\left(\left(|\mathbf{u}|^{2}-1\right) \mathbf{u}, \mathbf{R}_{h}(\mathbf{u})-\mathbf{U}_{n}\right) \\
=: & T_{11}^{n}+\ldots+T_{16}^{n} .
\end{aligned}
$$

By Step 2, $T_{12}^{n}+T_{13}^{n}+T_{14}^{n}=0$. Therefore

$$
\gamma k_{\varepsilon}\left\|\nabla\left(\mathbf{u}-\mathbf{U}_{n}\right)\right\|_{L^{2}}^{2} \leqslant T_{11}^{n}+T_{15}^{n}+T_{16}^{n}+T_{5}^{n}+T_{6}^{n}+T_{7}^{n} .
$$

All of the above is true for any $n \in \mathbb{N}$. Now, consider the limit $n \rightarrow+\infty$ and $h \rightarrow 0$. Note that, by a density argument,

$$
\left\|\mathbf{R}_{h}(\mathbf{u})-\mathbf{U}_{n}\right\|_{X} \leqslant\left\|\mathbf{R}_{h}(\mathbf{u})-\mathbf{u}\right\|_{X}+\left\|\mathbf{u}-\mathbf{U}_{n}\right\|_{X} \underset{n \rightarrow+\infty}{\stackrel{h \rightarrow 0}{\longrightarrow}} 0
$$

for $X=H^{1}$ and, by embedding, for $X=L^{p}(p<6)$. Therefore, similarly to Step 2, the terms $T_{5}^{n}, T_{6}^{n}, T_{7}^{n}, T_{15}^{n}$ and $T_{16}^{n}$ all vanish in the limit $h \rightarrow 0$ and $n \rightarrow+\infty$. Finally, $T_{11}^{n}$ vanishes using Lemma 5.6, as in Step 2, and the $H^{1}$-stability of the Ritz projection.

Step 4: solves equation (5.3), and $0 \leqslant s \leqslant 1$. Let $\varphi \in C^{\infty}(\Omega)$ be fixed, $n \in \mathbb{N}$, and $h>0$. Set

$$
2 \alpha \varepsilon(\nabla s, \nabla \varphi)+\gamma\left(|\nabla \mathbf{u}|^{2} s, \varphi\right)+\frac{\alpha}{2 \varepsilon}(s-1, \varphi)=: 2 \alpha \varepsilon T_{1}+\gamma T_{2}+\frac{\alpha}{2 \varepsilon} T_{3} .
$$

We have

$$
T_{1}=\left(\nabla S_{n}, \nabla \mathcal{I}_{h}(\varphi)\right)+\left(\nabla S_{n}, \nabla\left(\varphi-\mathcal{I}_{h}(\varphi)\right)\right)+\left(\nabla\left(s-S_{n}\right), \nabla \varphi\right)=: T_{11}^{n}+T_{12}^{n}+T_{13}^{n},
$$

with $T_{12}^{n}, T_{13}^{n} \rightarrow 0$ by the strong $H^{1}$ convergence of $\mathcal{I}_{h}(\cdot)$ and the weak $H^{1}$ convergence of $S_{n}$, respectively, as in Step 2. Also,

$$
\begin{aligned}
T_{2}= & \left(\left|\nabla \mathbf{U}_{n}\right|^{2} S_{n}, \mathcal{I}_{h}(\varphi)\right)+\left(\left|\nabla \mathbf{U}_{n}\right|^{2} S_{n}, \varphi-\mathcal{I}_{h}(\varphi)\right) \\
& +\left(\left(|\nabla \mathbf{u}|^{2}-\left|\nabla \mathbf{U}_{n}\right|^{2}\right) S_{n}, \varphi\right)+\left(|\nabla \mathbf{u}|^{2}\left(s-S_{n}\right), \varphi\right) \\
= & : T_{21}^{n}+T_{22}^{n}+T_{23}^{n}+T_{24}^{n},
\end{aligned}
$$

with $T_{22}^{n}, T_{23}^{n}, T_{24}^{n} \rightarrow 0$ by the properties of the Lagrange interpolation, Step 3, and Lemma 5.6, respectively. Finally,

$$
\begin{aligned}
T_{3}= & \left(S_{n}-1, \mathcal{I}_{h}(\varphi)\right)_{h}+\left(S_{n}-1, \mathcal{I}_{h}(\varphi)\right)-\left(S_{n}-1, \mathcal{I}_{h}(\varphi)\right)_{h} \\
& +\left(S_{n}-1, \varphi-\mathcal{I}_{h}(\varphi)\right)+\left(s-S_{n}, \varphi\right) \\
= & : T_{31}^{n}+\cdots+T_{35}^{n},
\end{aligned}
$$

with $\left|T_{32}^{n}+T_{33}^{n}\right| \leqslant C h\left\|\nabla S_{n}\right\|_{L^{2}(\Omega)}\left\|\mathcal{I}_{h}(\varphi)\right\|_{L^{2}(\Omega)} \rightarrow 0$, and $T_{34}^{n}, T_{35}^{n} \rightarrow 0$ by the strong $L^{p}$ convergence of $\mathcal{I}_{h}(\cdot)$ and $S_{n}$, respectively. 
So, putting all of the above together, we have, for $n \in \mathbb{N}$ and $h>0$ fixed,

$$
2 \alpha \varepsilon(\nabla s, \nabla \varphi)+\gamma\left(|\nabla \mathbf{u}|^{2} s, \varphi\right)+\frac{\alpha}{2 \varepsilon}(s-1, \varphi)=: 2 \alpha \varepsilon T_{13}^{n}+\gamma T_{21}^{n}+\frac{\alpha}{2 \varepsilon} T_{31}^{n}+T^{n},
$$

where $2 \alpha \varepsilon T_{13}^{n}+\gamma T_{21}^{n}+\frac{\alpha}{2 \varepsilon} T_{31}^{n}=0$ by construction. Now, letting $n \rightarrow+\infty$ and $h \rightarrow 0$, we get $T^{n} \rightarrow 0$, as shown above.

By a density argument, $s$ solves equation (5.3) for $\varphi \in H^{1}(\Omega) \cap L^{\infty}(\Omega)$. And since replacing $s$ pointwise by $0 \vee s \wedge 1$ would only decrease every term of this energy, $0 \leqslant s \leqslant 1$ follows.

REMARK 5.7 For $d \leqslant 2$, one can also get $\nabla S_{n} \rightarrow \nabla s$ strongly in $L^{2}\left(\Omega ; \mathbb{R}^{m}\right)$, with an argument similar to Step 3, using the equations for $S_{n}$ and $s$ and a test function $R_{h}(s)-S_{n}$. This breaks down for $d \geqslant 3$ because of the lack of $L^{\infty}$-stability of the Ritz projection.

\section{Computational studies}

To implement Algorithm 5.2, we use a simple fixed-point strategy (with three iterations) for the Ginzburg-Landau term.

To process real images, we suggest to amend Ambrosio and Tortorelli's energy to $A T_{\varepsilon}(\mathbf{u}, v, s)$ : $H^{1}\left(\Omega, \mathbb{S}^{m-1}\right) \times H^{1}(\Omega) \times H^{1}(\Omega) \rightarrow[0,+\infty]$,

$$
\begin{aligned}
A T_{\varepsilon}(\mathbf{u}, v, s):= & \frac{\gamma}{2} \int_{\Omega}\left(s^{2}+k_{\varepsilon}\right)|\nabla \mathbf{u}|^{2} \mathrm{~d} \mathbf{x}+\frac{\lambda}{2} \int_{\Omega}|\mathbf{u}-\mathbf{g}|^{2} \mathrm{~d} \mathbf{x} \\
& +\frac{\gamma_{1}}{2} \int_{\Omega}\left(s^{2}+k_{\varepsilon}\right)|\nabla v|^{2} \mathrm{~d} \mathbf{x}+\frac{\lambda_{1}}{2} \int_{\Omega}|v-b|^{2} \mathrm{~d} \mathbf{x} \\
& +\alpha \int_{\Omega}\left(\varepsilon|\nabla s|^{2}+\frac{1}{4 \varepsilon}(1-s)^{2}\right) \mathrm{d} \mathbf{x},
\end{aligned}
$$

with $\gamma, \gamma_{1}, \alpha, \lambda, \lambda_{1}$ positive constants and $b, v \in L^{\infty}(\Omega) \cap H^{1}(\Omega)$ the brightness components of the original and the processed image, respectively (normalised to lie in $[0,1]$ ). So, we add a smoothing and a fidelity term for the brightness component in the second line of (6.1). The idea here is that the smoothing term for the chromaticity component forces $|s|$ to be small whenever $|\nabla \mathbf{u}|$ is large, while the smoothing term for the brightness component does the same whenever $|\nabla v|$ is large. So we expect $\{s \approx 0\}$ to approximate the union of the essential jump sets of the chromaticity and the brightness component.

This necessitates the adaptation of the optimisation problem for $s$ as well as the solution of a third optimisation problem, which we place between the two existing ones.

If we process an image with more noise in the chromaticity than in the brightness, as is usually the case with images from digital cameras, we can now choose to give more weight to the information on the brightness component, and the chromaticity component will profit from the better information on the brightness component through the joint edge set, as illustrated in Example 4.

\subsection{Academic images, Splitting \& Projection}

All arrows below are scaled in length to fit the plots. What we call $h$ below is the length of the two shorter sides of the rectangular triangles in our triangulations, i.e., it is shorter than the actual diameter of the triangles (by a factor of $\sqrt{2}$ ). 
EXAmple 1 Let $\Omega:=(0,1)^{2}$ and $\mathbf{G}$ as in the left plot in Figure 2. The right picture shows a section along $x=0.5$, where the $z$-values of the two regions are the closest. We use a triangulation consisting of $2^{2 * 8}$ halved squares (along the direction $(1,1)$ ), i.e., 131072 triangles, with 66049 nodes, and $h=2^{-8} \approx 4 * 10^{-3}$. The initial values for $\mathbf{U}$ and $S$ are $\mathbf{U}_{0} \equiv \mathbf{G}$ and $S_{0} \equiv 0.5$, respectively. We test two different values for $\epsilon$, and choose the remaining parameters by experiment:

(1) $\varepsilon=5 * h \approx 2 * 10^{-2}, \gamma=1, \alpha=0.7, \lambda=60$, and $k_{\varepsilon}=10^{-6}$.

(2) $\varepsilon=h / 6 \approx 6 * 10^{-4}, \gamma=1.2, \alpha=0.5, \lambda=2 * 10^{3}$, and $k_{\varepsilon}=10^{-6}$.

Figure 2 shows the initial values, Figure 3 the results after 10 iterations of our proposed algorithm. Figure 4 shows the detected edge sets and Figure 5 the Ambrosio-Tortorelli energies over time. In our analysis, we did the discretisation limit first, and the $\Gamma$-limit afterwards, which would suggest choosing $\varepsilon$ larger than $h$. Interestingly, however, the algorithm remains stable for significantly smaller $\varepsilon$ (this is true for both algorithms). And while the qualitative differences in the image are minimal, a larger phase parameter obviously leads to a less precise edge set.

The next example numerically studies blowup behaviour for the $W^{1, \infty}$-norm of the iterates $\left\{\mathbf{U}_{n}, S_{n}\right\}$ in the absence of a fidelity term, i.e., for $\lambda=0$. This is motivated by blowup results for harmonic maps (to the sphere); see e.g. [46-49, 37, 6]. In particular, it is known that for $d=2$, singularities only appear for large initial energy. And any harmonic map (for general $d$ ) is smooth outside a set whose $(d-2)$-dimensional Hausdorff measure is zero (see [45, 46, 38, 29, 9, 41, 40]).

EXAMPLE 2 Let $\Omega$ be as above. We first use a triangulation consisting of $2^{2 * r}, r=8$, halved squares as above, and later use coarser ones $(r \in\{5, \ldots, 8\})$ for comparison. Let $\gamma=1=\alpha$, $\lambda=0, \varepsilon=5 * h$, and $k_{\varepsilon}=10^{-6}$. We use initial data for $\mathbf{U}$ and $S$ as shown in Figure 6 (leftmost column): $\mathbf{U}_{0}$ is constantly $(0,0,1)$ in the periphery of the image, $(0,0,-1)$ at the centre, and varying continuously inside a circle around the centre; as well as $S=0$ at the centre, $S=1$ in the periphery, and smoothly varying in between.

Figure 6 shows iterates $n \in\{0,3,5\}$ for $r=8$ (crops in the case of $\mathbf{U}_{n}$ ), Figure 7 shows the total energy for $r \in\{5, \ldots, 8\}$, while Figure 8 shows the $W^{1, \infty}$-norms of $\mathbf{U}_{n}$ and $S_{n}$ for $r \in$ $\{5, \ldots, 8\}$, which both show blowup behaviour. This time it is $\mathbf{U}_{n}$ which appears one step ahead of $S_{n}$ with respect to blowup behaviour. Depending somewhat on $r$, the system matrices become close to singular after 6-7 iterations, so after this point, the results can no longer be expected to be reliable. The arrow at the centre of $\mathbf{U}$ at this point still points down, while the rest of $\mathbf{U}$ points up. The variable $S$, on the other hand, becomes 1 everywhere, except for the centre, where it stays 0 . After breakdown, the arrows move erratically, but perfectly synchronised with one another.

\subsection{Academic images, Penalisation \& Splitting}

The next example studies the same setting as Example 1, this time with Algorithm 5.2, i.e., the sphere constraint is enforced by penalisation instead of projection. Again, all arrows are scaled in length to fit the plots.

ExAmple 3 The setting is as in Example 1. Parameters are $\gamma=1.2, \alpha=0.5, \lambda=2 * 10^{3}$, $\varepsilon=10^{-3}, k_{\varepsilon}=10^{-6}$, and $\delta_{\varepsilon}=0.1$ (chosen by experiment).

The result $\mathbf{U}$ after 10 iterations looks just as in Example 1 (Figure 3), so we omit the corresponding figures. The detected edge set after 10 iterations, however, is less exact, as shown in 
$\mathrm{u}_{0}$

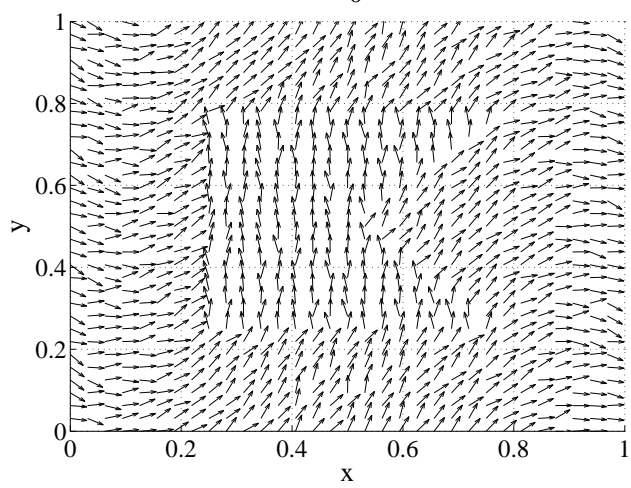

$\mathrm{u}_{0}(5.000000 \mathrm{e}-01,:)_{\mathrm{z}}$

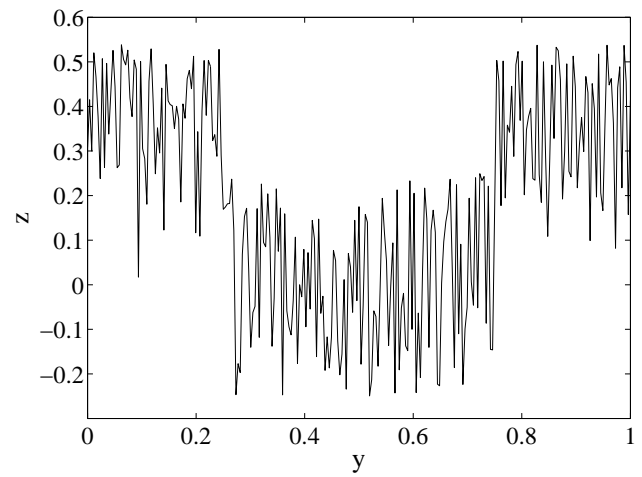

FIG. 2. Example 1: Original image (left) and $z$-values of a vertical section through it $(x=0.5$, right).
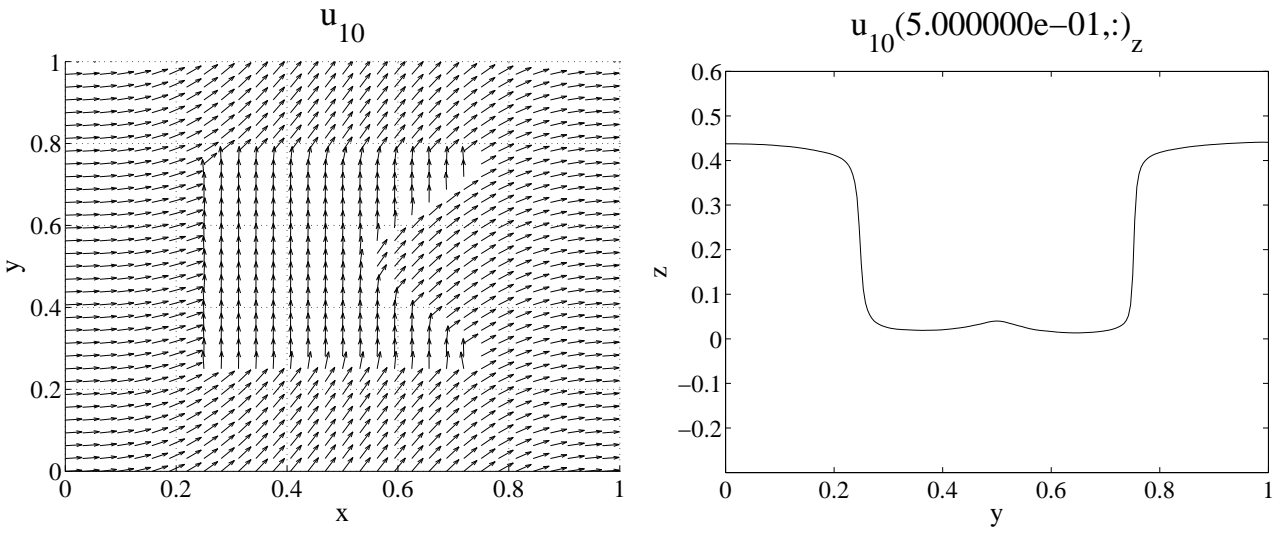

$\mathrm{u}_{10}$

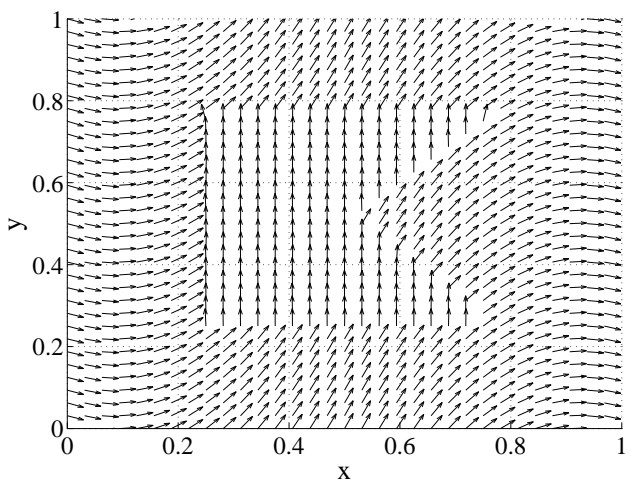

$\mathrm{u}_{10}(5.000000 \mathrm{e}-01,:)$

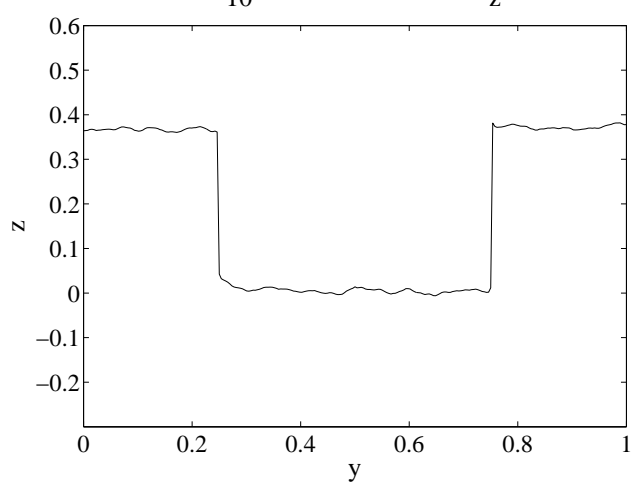

FIG. 3. Example 1: Image (left) and section (right) after 10 iterations for $\varepsilon=5 * h$ (top) and $\varepsilon=h / 6$ (bottom). 

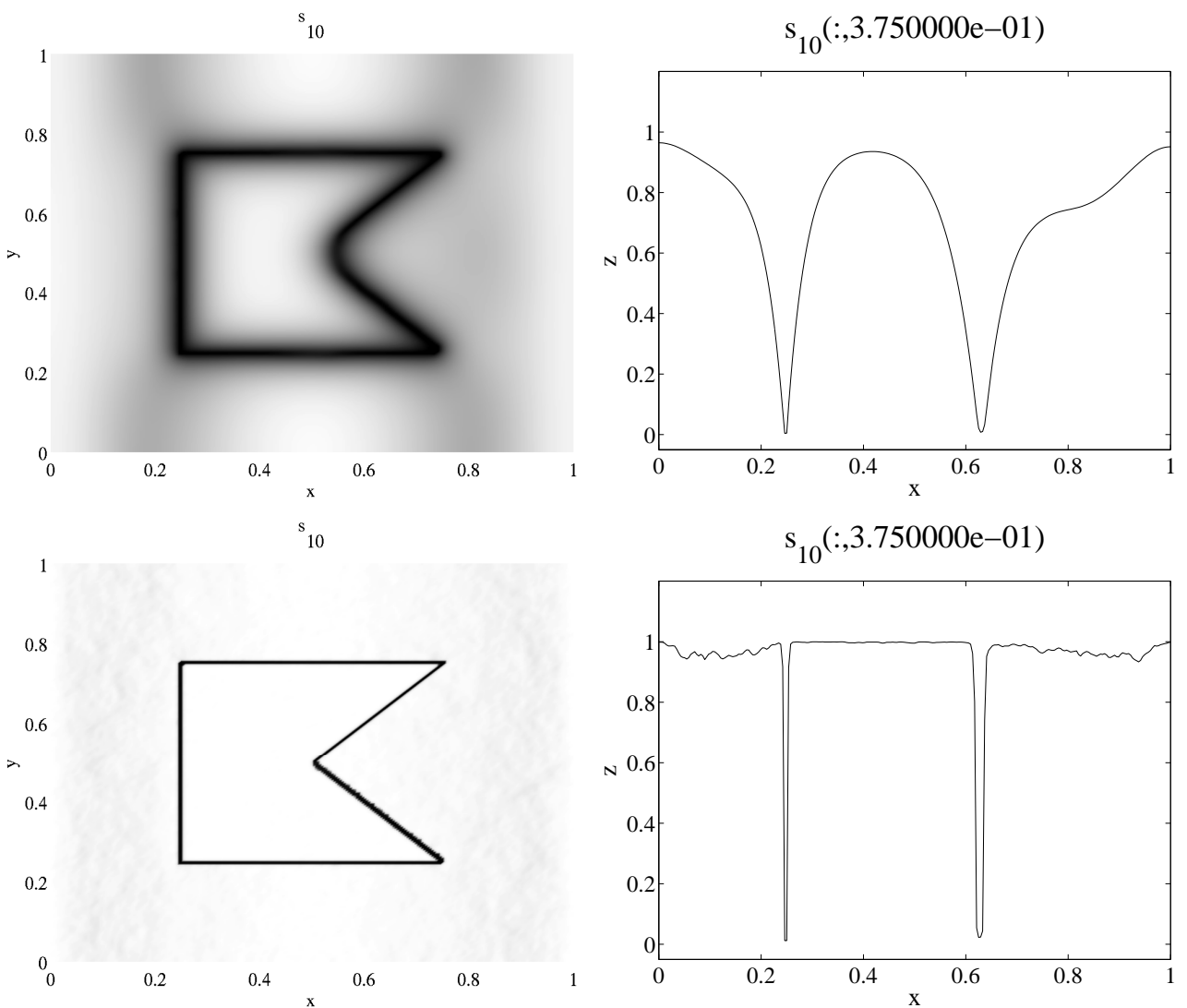

FIG. 4. Example 1: Edge set (left) and horizontal section through it ( $y=0.375$, right) after 10 iterations for $\varepsilon=5 * h$ (top) and $\varepsilon=h / 6$ (bottom).
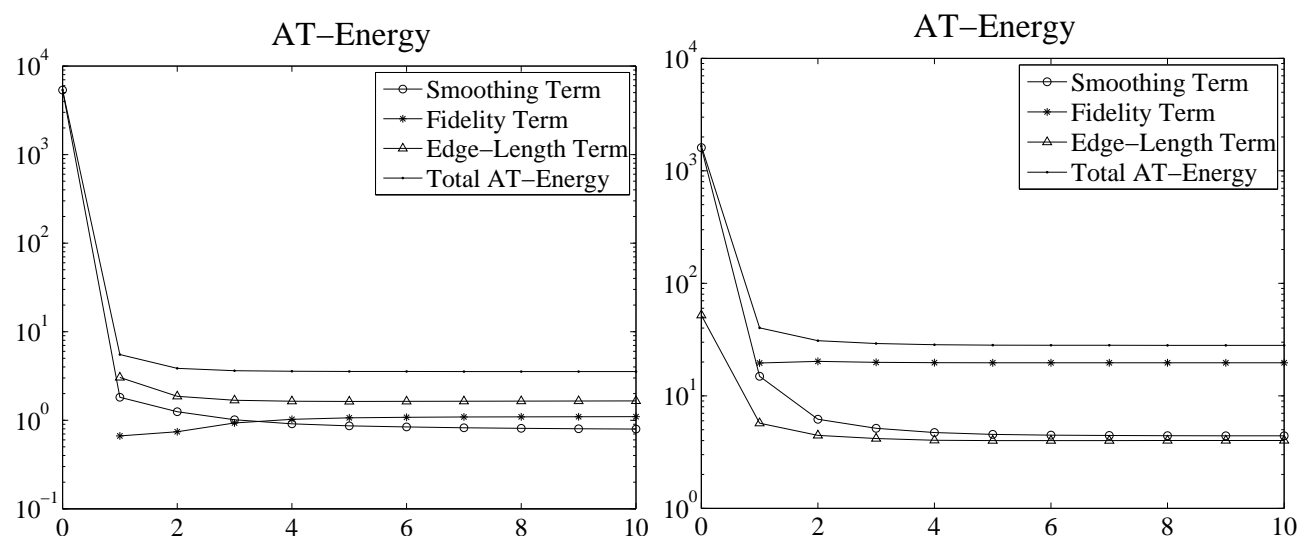

FIG. 5. Example 1: Ambrosio-Tortorelli energy (10 iterations, logarithmic plot) for $\varepsilon=5 * h$ (left) and $\varepsilon=h / 6$ (right). 

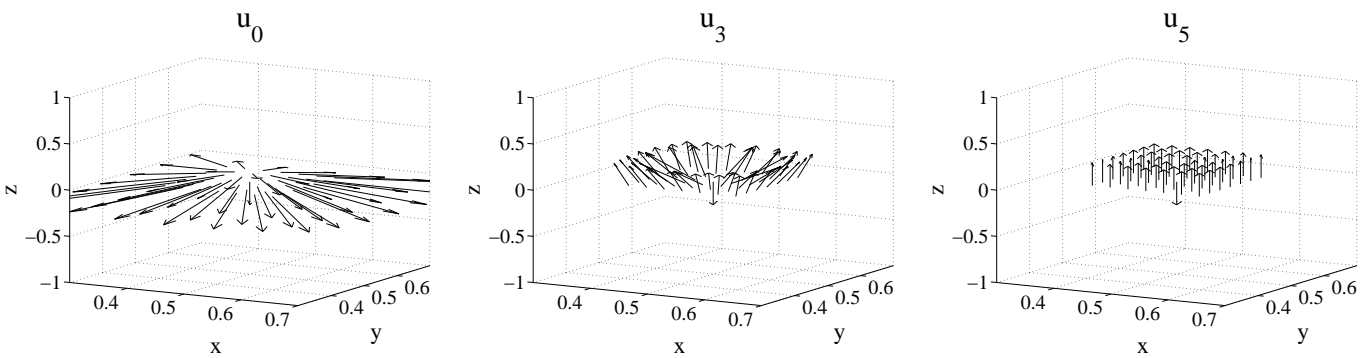

FIG. 6. Example 2: Detail of $\mathbf{U}_{n}$ (top) and full image of $S_{n}$ (bottom) for $n \in\{0,3,5\}$.
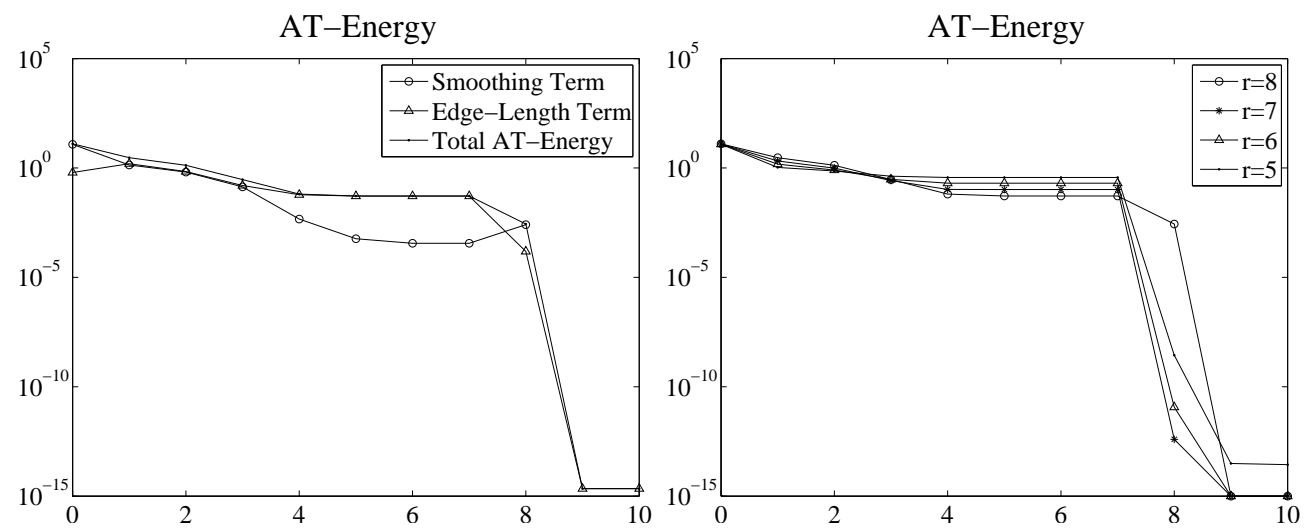

FIG. 7. Example 2: Ambrosio-Tortorelli energy, 10 steps, for $r=8$ (left) and $r \in\{5, \ldots, 8\}$ (right), $y$-logarithmic plots. 

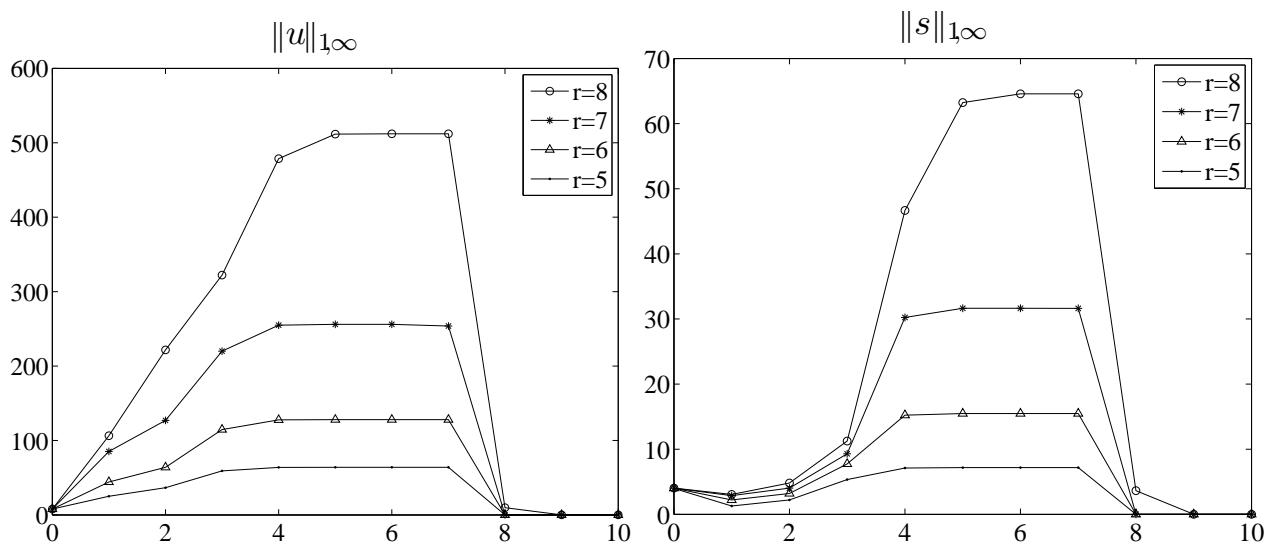

FIG. 8. Example 2: $W^{1, \infty}$-norm of $\mathbf{U}$ and $S$ for $r \in\{5, \ldots, 8\}$.
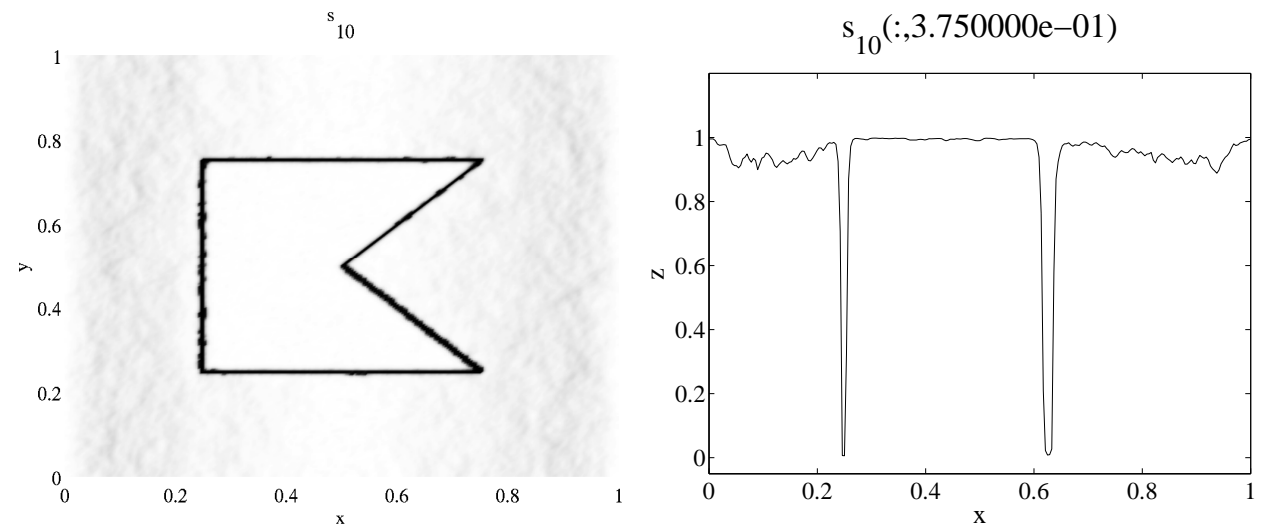

FIG. 9. Example 3: Edge set (left) and horizontal section through it ( $y=0.375$, right) after 10 iterations.
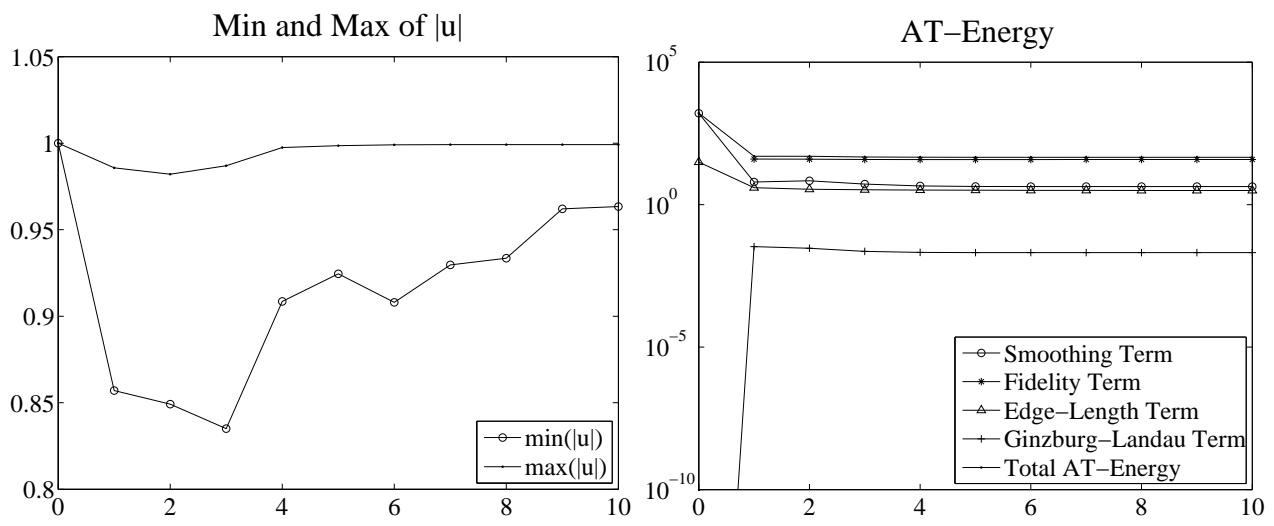

FIG. 10. Example 3: min and max of $|\mathbf{U}|$ (left), and Ambrosio-Tortorelli energy (right) for 10 iterations. 
Figure 9. Figure 10 shows the global minimum and maximum of $|\mathbf{U}|$ and the Ambrosio-Tortorelli energy over time.

For $\delta_{\varepsilon}$ between about $5 * 10^{-3}$ and at least $10^{2}$, the results are qualitatively very similar to the ones in Example 1, but the detected edge set is less exact, and $|\mathbf{U}|$ can be quite a bit shorter than 1 . For $\delta_{\varepsilon}$ smaller than $5 * 10^{-3}$ (which would be advantageous for the accuracy of $|\mathbf{U}|$ ), the results break down, which is in accordance with our theoretical results.

\subsection{Real image, Splitting \& Projection}

EXAMPLE 4 We try our algorithm on a small photograph $(399 \times 299$ pixels $)$, as shown in Figure 11 . We choose $\Omega:=(0,399 / 299) \times(0,1)$, whence $h=1 / 298 \approx 3 * 10^{-3}$, the pixels are used as nodes, each square of four pixels giving rise to two triangles. We further choose $S_{0} \equiv 1$ and add two different kinds of noise to the image:

(1) RGB noise: $R=R_{0}+0.3 *$ randn, and $G$ and $B$ analogously, where randn are pseudo-random values drawn from the standard normal distribution. After this operation, we crop $R, G$, and $B$ to lie in $[0,1]$ (where $R_{0}, G_{0}, B_{0}$ were scaled to lie). This is shown in Figure 11.

(2) $\mathrm{CB}$ noise, mainly in the chromaticity component: $\mathbf{C}=\mathbf{C}_{0}+0.5 * \operatorname{randn} * \mathbf{C}_{0} \times[1,1,1]$, and $B=B_{0}+0.01 *$ randn. After this operation, $\mathbf{C}$ is projected onto the sphere, and $B$ is cropped to lie in $[0,1]$. This is shown in Figure 14.

Our CB algorithm was in both cases compared to a channelwise RGB computation for the same image, with all channels sharing the same edge set. Parameters were chosen as follows (by experiment):

(1) RGB computation: $\alpha=0.3, \beta=10^{-2}, \gamma=10^{3}, \varepsilon=10^{-4}$, and $k_{\varepsilon}=10^{-7}$.

CB computation: $\alpha=\alpha_{1}=0.5, \beta=8 * 10^{-3}, \gamma=\gamma_{1}=10^{3}, \varepsilon=10^{-4}$, and $k_{\varepsilon}=10^{-7}$.

(2) RGB computation: $\alpha=0.5, \beta=5 * 10^{-3}, \gamma=50, \varepsilon=10^{-4}$, and $k_{\varepsilon}=10^{-7}$.

CB computation: $\alpha=\alpha_{1}=0.3, \beta=5 * 10^{-2}, \gamma=10^{2}, \gamma_{1}=5 * 10^{5}, \varepsilon=10^{-4}$, and $k_{\varepsilon}=10^{-7}$.
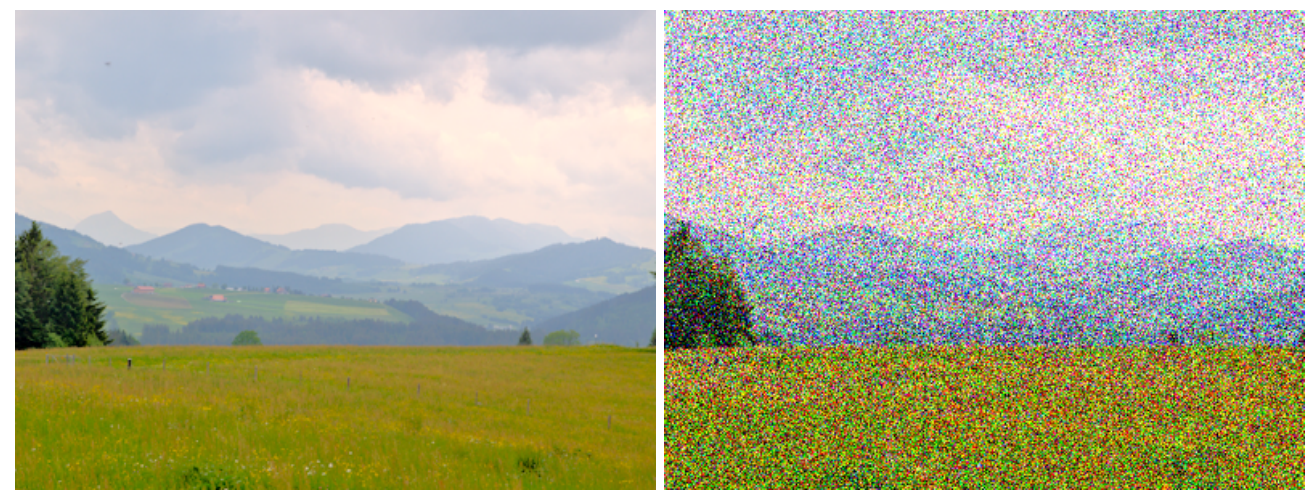

FIG. 11. Example 4.1: Original image (left) and image with RGB noise (right). 

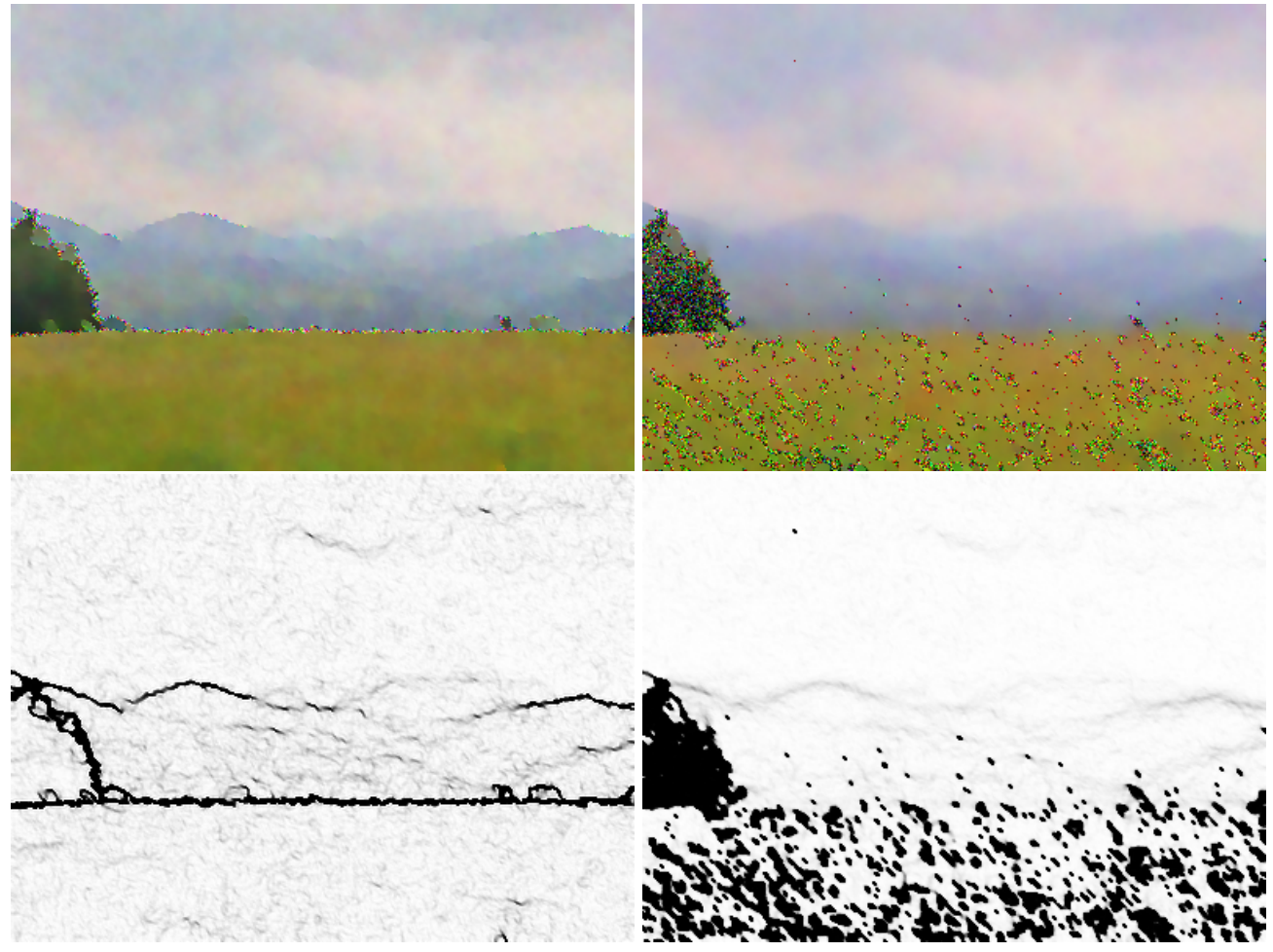

FIG. 12. Example 4.1: Image (top) and edge set (bottom) after 10 iterations; for RGB (left) and CB (right).
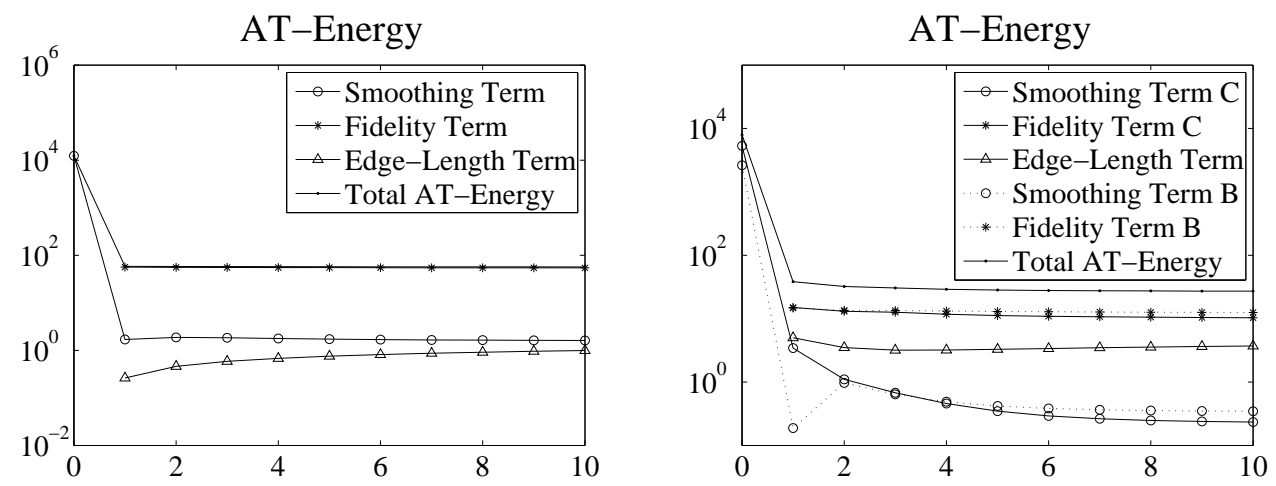

FIG. 13. Example 4.1: Expanded Ambrosio-Tortorelli energy (10 iterations, $y$-logarithmic plots), RGB (left) and CB (right). 
APPROXIMATIONS OF MUMFORD-SHAH FUNCTIONAL
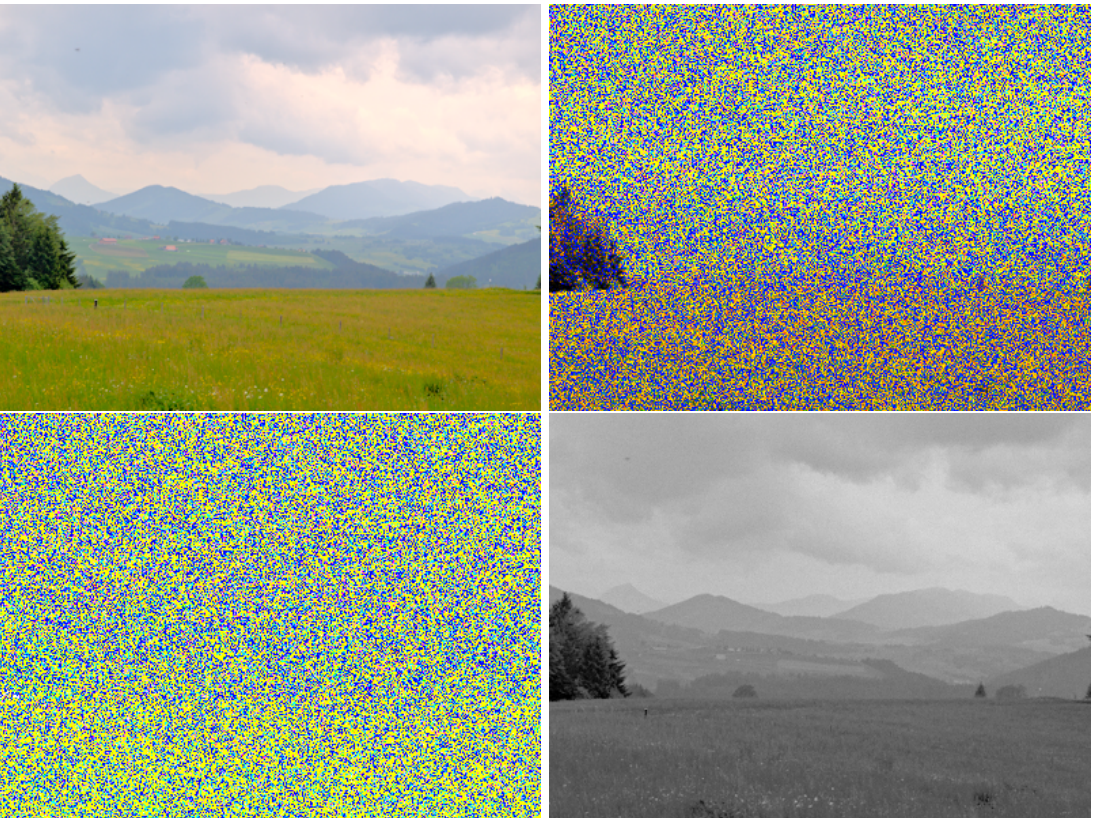

FIG. 14. Example 4.2: Original image and image with CB noise (top), as well as noisy chromaticity (bottom left) and brightness (bottom right) components.
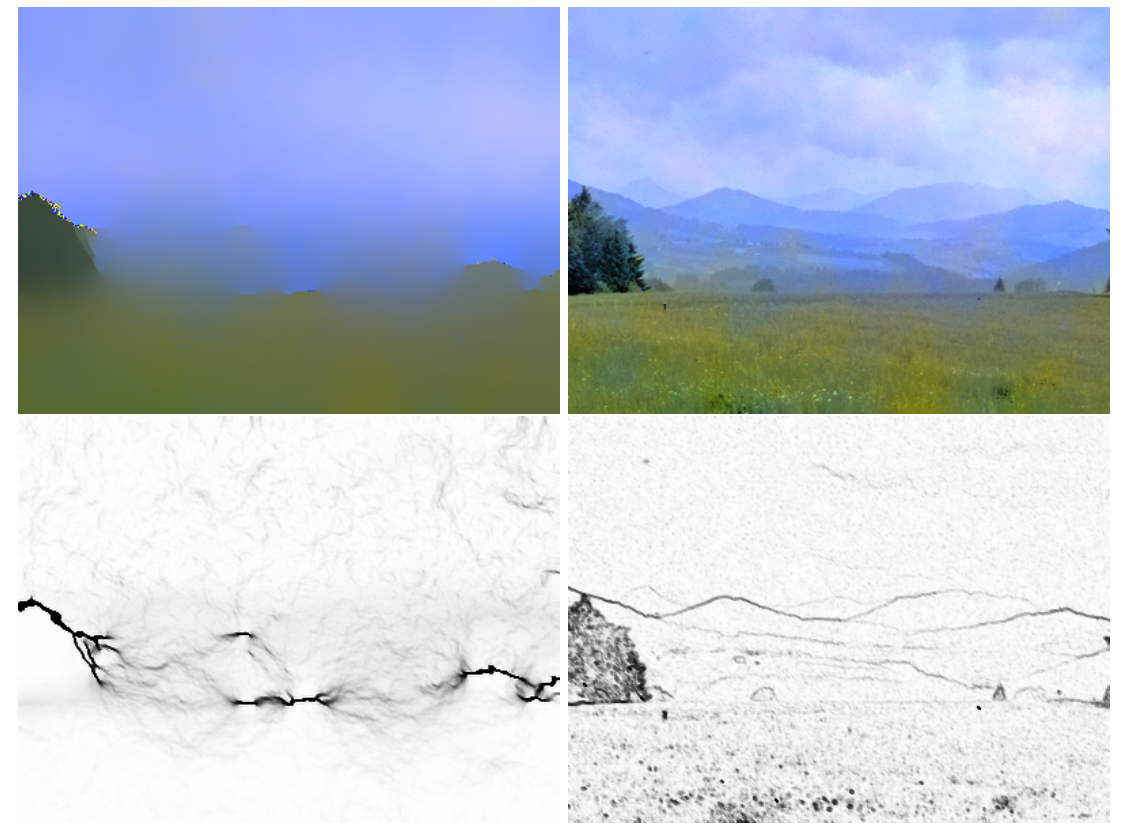

FIG. 15. Example 4.2: Image (top) and edge set (bottom) after 10 iterations; for RGB (left) and CB (right). 

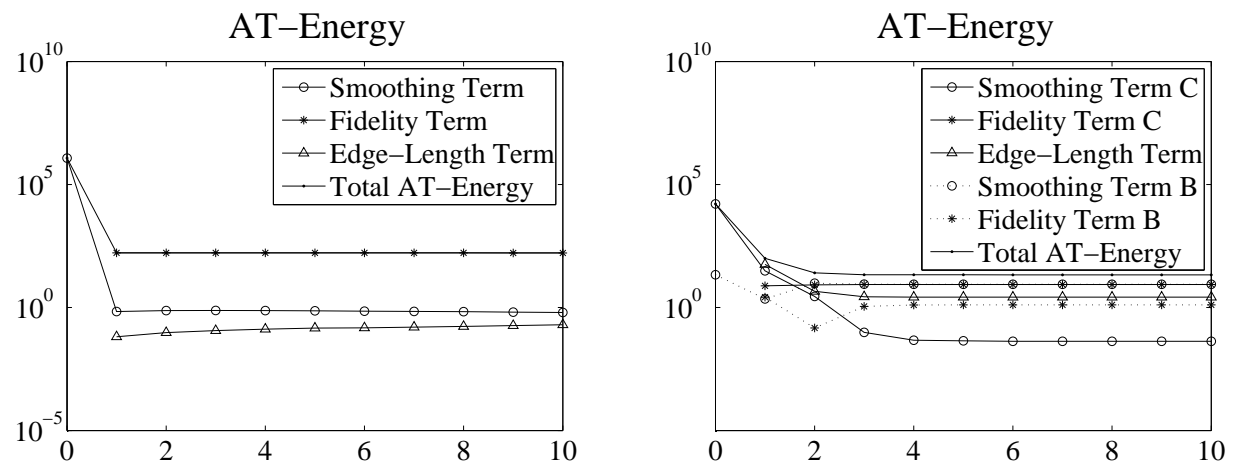

FIG. 16. Example 4.2: Expanded Ambrosio-Tortorelli energy (10 iterations, $y$-logarithmic plots), RGB (left) and CB (right).

First, let us look at the computations with RGB noise: Figure 11 shows the noisy initial image, and Figure 12 the images and edge sets after 10 iterations. Figure 13 shows the expanded AmbrosioTortorelli energy over time. The energy terms labelled “...C”' belong to the chromaticity component, those labelled "... B" to the brightness. The channelwise RGB algorithm has the advantage here.

Next, let us look at the image with $\mathrm{CB}$ noise: Figure 14 shows the noisy initial image, and Figure 15 the images and edge sets after 10 iterations. Figure 16 shows the expanded AmbrosioTortorelli energy over time. The CB algorithm has a very clear advantage here.

\section{Acknowledgements}

The research presented in this paper was done as part of the author's PhD thesis [36] under the supervision of Andreas Prohl (Univ. Tübingen). Further thanks go to Sören Bartels (Univ. Bonn) for his help with the coding, and to Giovanni Bellettini (Univ. Roma), Ludwig Gauckler (Univ. Tübingen), Christoph Ortner (Oxford Univ.), Reiner Schätzle (Univ. Tübingen), and Markus Schmuck (MIT Cambridge, MA) for helpful discussions.

\section{REFERENCES}

1. Alouges, F. A new algorithm for computing liquid crystal stable configurations: the harmonic mapping case. SIAM J. Numer. Anal. 34 (1997), 1708-1726. Zbl 0886.35010 MR 1472192

2. Ambrosio, L., Fusco, N., \& Pallara, D. Functions of Bounded Variation and Free Discontinuity Problems. Oxford Math. Monogr., Oxford Univ. Press, New York (2000). Zbl 0957.49001 MR 1857292

3. Ambrosio, L., \& Tortorelli, V. M. Approximation of functionals depending on jumps by elliptic functionals via $\Gamma$-convergence. Comm. Pure Appl. Math. 43 (1990), 999-1036. Zbl 0722.49020 MR 1075076

4. Ambrosio, L., \& Tortorelli, V. M. On the approximation of free discontinuity problems. Boll. Un. Mat. Ital. B (7) 6 (1992), 105-123. Zbl 0776.49029 MR 1164940

5. BARTELS, S. Stability and convergence of finite-element approximation schemes for harmonic maps. SIAM J. Numer. Anal. 43 (2005), 220-238. Zbl 1090.35014 MR 2177142

6. Bartels, S., \& Prohl, A. Constraint preserving implicit finite element discretization of harmonic map flow into spheres. Math. Comp. 76 (2007), 1847-1859. Zbl 1124.65089 MR 2336271 
7. Bartels, S., \& Prohl, A. Stable discretization of scalar and constrained vectorial Perona-Malik equation. Interfaces Free Bound. 9 (2007), 431-453. Zbl 1147.35011 MR 2358212

8. Bellettini, G., \& Coscia, A. Discrete approximation of a free discontinuity problem. Numer. Funct. Anal. Optim. 15 (1994), 201-224. Zbl 0806.49002 MR 1272202

9. Bethuel, F. On the singular set of stationary harmonic maps. Manuscripta Math. 78 (1993), 417-443. Zbl 0792.53039 MR 1208652

10. Bourdin, B. Image segmentation with a finite element method. Math. Model. Numer. Anal. 33 (1999), 229-244. Zbl 0947.65075 MR 1700033

11. Braides, A. Approximation of Free-Discontinuity Problems. Lecture Notes in Math. 1694, Springer, Berlin (1998). Zbl 0909.49001 MR 1651773

12. Braides, A. $\Gamma$-convergence for Beginners. Oxford Lecture Ser. Math. Appl. 22, Oxford Univ. Press, Oxford (2002). Zbl 1198.49001 MR 1968440

13. Braides, A., \& Dal Maso, G. Non-local approximation of the Mumford-Shah functional. Calc. Var. Partial Differential Equations 5 (1997), 293-322. Zbl 0873.49009 MR 1450713

14. Brenner, S. C., \& ScotT, L. R. The Mathematical Theory of Finite Element Methods. 2nd ed., Texts Appl. Math. 15, Springer, New York (2002). Zbl 1012.65115 MR 1894376

15. BURKe, S., ORtNer, C., \& SÜLI, E. An adaptive finite element approximation of a variational model of brittle fracture. SIAM J. Numer. Anal. 48 (2010), 980-1012. Zbl pre05876979 MR 2669398

16. Calderer, M. C., Golovaty, D., Lin, F.-H., \& LiU, C. Time evolution of nematic liquid crystals with variable degree of orientation. SIAM J. Math. Anal. 33 (2002), 1033-1047. Zbl 1003.35108 MR 1897700

17. Carriero, M., \& Leaci, A. $S^{k}$-valued maps minimizing the $L^{p}$-norm of the gradient with free discontinuities. Ann. Scuola Norm. Sup. Pisa Cl. Sci. (4) 18 (1991), 321-352. Zbl 0753.49018 MR 1145314

18. Chambolle, A. Inverse problems in image processing and image segmentation: some mathematical and numerical aspects. In: C. E. Chidume (ed.), Mathematical Problems in Image Processing, ICTP, December 2000. ICTP Lecture Notes Series, Vol. 2; http://publications.ictp.it/lns/vol2.html.

19. Chan, T. F., KANG, S. H., \& SHEN, J. Total variation denoising and enhancement of color images based on the CB and HSV color models. J. Visual Comm. Image Rep. 12 (2001), 422-435.

20. Clément, P. Approximation by finite element functions using local regularization. Rev. Française Automat. Informat. Recherche Opérationnelle Sér. RAIRO Analyse Numérique 9 (1975), 77-84. Zbl 0368.65008 MR 0400739

21. Cohen, R., Lin, S. Y., \& Luskin, M. Relaxation and gradient methods for molecular orientation in liquid crystals. Comput. Phys. Comm. 53 (1989), 455-465. MR 1004704

22. Cortesani, G. A finite element approximation of an image segmentation problem. Math. Models Methods Appl. Sci. 9 (1999), 243-259. Zbl 0937.65072 MR 1674564

23. Dal Maso, G. An Introduction to $\Gamma$-convergence. Progr. Nonlinear Differential Equations Appl. 8, Birkhäuser Boston, Boston, MA (1993). Zbl 0816.49001 MR 1201152

24. De Giorgi, E. Free discontinuity problems in calculus of variations. In: Frontiers in Pure and Applied Mathematics, North-Holland, Amsterdam (1991), 55-62. Z Zbl 0758.49002 MR 1110593

25. DE GIORgI, E. Variational free-discontinuity problems. In: International Conference in Memory of Vito Volterra (Rome, 1990), Atti Convegni Lincei 92, Accad. Naz. Lincei, Rome (1992), 133-150. Zbl 1039.49507 MR 1783032

26. De Giorgi, E., \& Ambrosio, L. New functionals in the calculus of variations. Atti Accad. Naz. Lincei Rend. Cl. Sci. Fis. Mat. Natur. (8) 82 (1988), 199-210. Zbl 0715.49014 MR 1152641

27. De Giorgi, E., Carriero, M., \& Leaci, A. Existence theorem for a minimum problem with free discontinuity set. Arch. Ration. Mech. Anal. 108 (1989), 195-218. Zbl 0682.49002 MR 1012174

28. Dibos, F., \& SÉRÉ, E. An approximation result for the minimizers of the Mumford-Shah functional. Boll. Un. Mat. Ital. A (7) 11 (1997), 149-162. Zbl 0873.49008 MR 1438364 
29. Evans, L. C. Partial regularity for stationary harmonic maps into spheres. Arch. Ration. Mech. Anal. 116 (1991), 101-113. Zbl 0754.58007 MR 1143435

30. Evans, L. C., \& Gariepy, R. F. Measure Theory and Fine Properties of Functions. Stud. Adv. Math., CRC Press, Boca Raton, FL (1992). Zbl 0804.28001 MR 1158660

31. Federer, H. Geometric Measure Theory. Grundlehren Math. Wiss. 153, Springer, New York (1969). Zbl 0176.00801 MR 0257325

32. FOCARDI, M. On the variational approximation of free-discontinuity problems in the vectorial case. Math. Models Methods Appl. Sci. 11 (2001), 663-684. Zbl 1010.49010 MR 1832998

33. FoCARDI, M. Variational approximation of vectorial free discontinuity problems: the discrete and continuous case. PhD thesis, Scuola Norm. Sup. Pisa (2002); http://cvgmt.sns.it/papers/foc01/.

34. Gilbarg, D., \& Trudinger, N. S. Elliptic Partial Differential Equations of Second Order. 2nd ed., Grundlehren Math. Wiss. 224, Springer, Berlin (1983). Zbl 0562.35001 MR 0737190

35. GiUsti, E. Minimal Surfaces and Functions of Bounded Variation. Monogr. Math. 80, Birkhäuser, Basel (1984). Zbl 0545.49018 MR 0775682

36. Haehnle, J. Numerical analysis of the Mumford-Shah and Mumford-Shah-Euler functionals for sphere-valued functions, and applications to numerical image processing. $\mathrm{PhD}$ thesis, Tübingen (2010).

37. HARdT, R. M. Singularities of harmonic maps. Bull. Amer. Math. Soc. (N.S.) 34 (1997), 15-34. Zbl 0871.58026 MR 1397098

38. HÉlEIN, F. Régularité des applications faiblement harmoniques entre une surface et une variété riemannienne. C. R. Acad. Sci. Paris Sér. I Math. 312 (1991), 591-596. Zbl 0728.35015 MR 1101039

39. LIN, F.-H. Nonlinear theory of defects in nematic liquid crystals; phase transition and flow phenomena. Comm. Pure Appl. Math. 42 (1989), 789-814. Zbl 0703.35173 MR 1003435

40. LIN, F.-H. Gradient estimates and blow-up analysis for stationary harmonic maps. Ann. of Math. (2) 149 (1999), 785-829. Z Zbl 0949.58017 MR 1709303

41. Lin, F. H., \& WANG, C. Y. Stable stationary harmonic maps to spheres. Acta Math. Sin. (Engl. Ser.) 22 (2006), 319-330. Zbl 1121.58017 MR 2214353

42. Lin, S. Y., \& LuSKin, M. Relaxation methods for liquid crystal problems. SIAM J. Numer. Anal. 26 (1989), 1310-1324. Zbl 0685.65058 MR 1025090

43. Mumford, D., \& SHAH, J. Optimal approximations by piecewise smooth functions and associated variational problems. Comm. Pure Appl. Math. 42 (1989), 577-685. Zbl 0691.49036 MR 0997568

44. OSHER, S. J., \& VESE, L. A. Numerical methods for $p$-harmonic flows and applications to image processing. SIAM J. Numer. Anal. 40 (2002), 2085-2104. Zbl 1035.65065 MR 1974176

45. Schoen, R., \& Uhlenbeck, K. A regularity theory for harmonic maps. J. Differential Geom. 17 (1982), 307-335. Z Zbl 0521.58021 MR 0664498

46. Schoen, R., \& Uhlenbeck, K. Regularity of minimizing harmonic maps into the sphere. Invent. Math. 78 (1984), 89-100. Zbl 0555.58011 MR 0762354

47. Struwe, M. On the evolution of harmonic mappings of Riemannian surfaces. Comment. Math. Helv. 60 (1985), 558-581. Z Zbl 0595.58013 MR 0826871

48. Struwe, M. Geometric evolution problems. In: Nonlinear Partial Differential Equations in Differential Geometry (Park City, UT, 1992), IAS/Park City Math. Ser. 2, Amer. Math. Soc., Providence, RI (1996), 257-339. Zbl 0847.58012 MR 1369591

49. Struwe, M. Variational Methods. 3rd ed., Ergeb Math. Grenzgeb. 34, Springer, Berlin (2000). Zbl 0939.49001 MR 2431434

50. TAng, B., SAPIRo, G. R., \& CASElles, V. Color image enhancement via chromaticity diffusion. IEEE Trans. Image Process. 10 (2001), 701-707. Zbl 1037.68792

51. Virga, E. G. Variational Theories for Liquid Crystals. Appl. Math. Math. Comput. 8, Chapman \& Hall, London (1994). Z Zbl 0814.49002 MR 1369095 